\title{
VISCOUS DAMAGE MODEL FOR TIMOSHENKO BEAM STRUCTURES
}

\author{
A. H. BARBAT, S. OLLER, E. OÑATE and A. HANGANU \\ Technical University of Catalonia, Edificio C1, Campus Norte UPC, Gran Capitán, s/n, \\ Barcelona 08034, Spain
}

(Received 20 December 1995 ; in revised form 26 November 1996)

\begin{abstract}
A local damage constitutive model based on Kachanov's theory is used within a finite element frame and applied to the case of 2D and 3D Timoshenko beam elements. The model takes into account viscous effects, thus allowing damping to be considered in a rigorous way. A damage index based on potential energy criteria, useful in evaluating the behaviour of structures or of parts of structures, is proposed. The procedure is applied to estimate the damage produced by seismic actions in reinforced concrete building structures, whose response is computed by using a non-linear Newmark-type incremental time integration scheme. Three numerical examples are included; one of them compares results obtained by using the proposed model with results of a laboratory test (C) 1997 Elsevier Science Ltd.
\end{abstract}

\section{INTRODUCTION}

In the case that a urban area is affected by a strong seismic motion, one of the most important problems is the evaluation of structural safety in that area, starting from the assessment of the actual damage experimented by structures. As an alternative, this evaluation can be performed by numerical simulation of the damage-related phenomena in the structures of that area. The specific type of structures considered in this paper are reinforced concrete buildings.

The structural damage will hereafter be defined as the degree of degradation that allows conclusions about the future capacity of a structure to withstand further loadings. It will be quantified through a damage index, which is a value of damage normalized to the failure level of the structure, so that a value equal to 1 will reflect complete structural failure.

Different definitions of global damage indices have been given in the literature for complex structures, generally based on a weighted average of the indices corresponding to different structural members (DiPasquale and Cakmak, 1989). Other works (Park et al., 1987) and Bracci et al. (1989) define a damage index for structural members using a linear combination between a ductility and an energy factor. In this paper, a global global damage index based on potential energy considerations is proposed. This index is formulated using a local damage constitutive model, based on Kachanov's theory (1958), and considering the influence of viscosity, thus including damping effects.

Kachanov's constitutive model has been chosen because it represents adequately the behaviour of the concrete subjected to monotonically increasing loads. Nevertheless, it is well-known that the basic Kachanov's theory has limitations concerning the closing of cracks during the unloading process. In the selection of the model have also been taken into account the high velocity of convergence and the simplicity of the assessment of its parameters. It is important to remember that the isotropic damage model considers the difference between the uniaxial tensional and compressional behaviour by means of the ratio of the uniaxial compression to the tension strengths. This implies that the shape of the uniaxial tension and compression curves is the same. Although this type of behaviour can be found in various geomaterials (Chen 1982; Oller 1988; Lubliner et al., 1989), it could be considered that for concrete it simplifies the real behaviour. The elasto-perfect plastic model of Von Mises has been chosen to describe the behaviour of the steel bars. Obviously, other constitutive models could be employed in characterizing the behaviour of 
both concrete and steel materials. However, the general methodology proposed in the paper is suitable whichever constitutive model is used.

In this paper, a structural model which applies these concepts to the analysis of beam structures is developed within the frame of the finite element method. Tangent and secant damping and stiffness matrices of the visco-damage constitutive law are deduced. Numerical examples showing the applicability of the proposed procedure are included.

\section{STRUCTURAL MODEL}

The structure is modelled using $C^{\circ}$ one dimensional finite elements based on Timoshenko's beam theory, generalized to $3 \mathrm{D}$. There is only a small difference between the constant shear strain distribution over the cross-section of the Timoshenko beam theory and the present one, consisting of the evaluation of $\gamma_{x y}(y, z)$ and $\gamma_{x z}(y, z)$. In this paper, the mean shear strains $\gamma_{x y}^{m}$ and $\gamma_{x z}^{m}$ corresponding to the direct Timoshenko beam formulation are corrected using Jourawski's stress distribution (Gere and Timoshenko, 1984) : $\dagger$

$$
\gamma_{x y}(y, z)=\gamma_{x y}^{m} A_{y}^{*}\left(\frac{S_{z}(y)}{J_{z} b(y)}\right), \quad \gamma_{x z}(y, z)=\gamma_{x z}^{m} A_{z}^{*}\left(\frac{S_{y}(z)}{J_{y} b(z)}\right)
$$

where $A_{y}^{*}=\chi_{y} A$ and $A_{z}^{*}=\chi_{z} A$ are the reduced cross-sections of $A$ and $\chi_{y}$ and $\chi_{z}$ are the stress distribution factors (Gere and Timoshenko, 1984). $b(y)$ and $b(z)$ are the width and the thickness of the cross-section, $J_{y}$ and $J_{z}$ are inertia moments and $S_{y}(z)$ and $S_{z}(y)$ are the statical moments with respect to the neutral axis of the upper and left portion of the crosssection, respectively. The proposed procedure, without providing the exact solution to the problem, assures an important improvement of the shear strain evaluation as compared

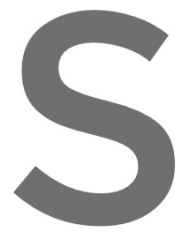
with Timoshenko's beam thoory.
The finite elements havd three ngdes and
fact that the constitutive model requires inform
discretization of the cross-section of the beam
discretization consists of layers [see Fig. I (a)].
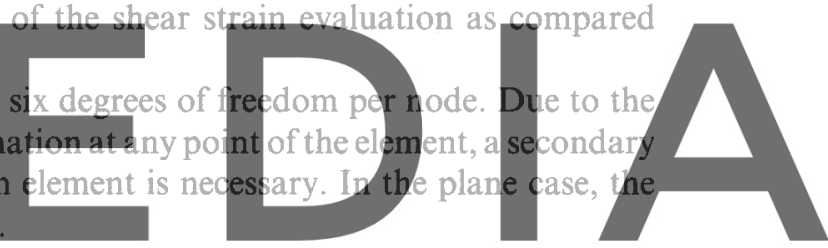

In the 3D case, the cross-section of the beam is discretized by means of an orthogonal

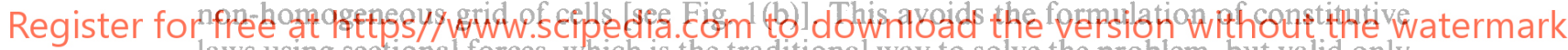
laws using sectional forces, which is the traditional way to solve the problem, but valid only in certain particular cases and having the additional drawback of lacking precision. The sectional forces are decomposed point by point, layer by layer, in stress tensors which are corrected by using the viscous damage model. The corrected sectional forces are subsequently obtained by integration over the section cells. These forces are then used to compute the residual forces, in order to iterate for equilibrium if necessary.

The relationships between the sectional variables of the problem and the variables corresponding to a certain point belonging to the mentioned section are described below. A local coordinate system is considered for the beam, its longitudinal axis $x$ forming a right triad with the other two axes. The sign convention for translations and rotations is the usual in classical mechanics. The displacement and strain fields are (Oñate, 1992)

$$
\begin{aligned}
\mathbf{u}(x, y, z) & =\mathbf{S}(y, z) \mathbf{u}_{o}(x) \\
\mathbf{\varepsilon}(x, y, z) & =\mathbf{S}(y, z) \hat{\boldsymbol{\varepsilon}}(x)
\end{aligned}
$$

where the variables have the following meaning: $\mathbf{u}=\{u, v, w\}^{\mathrm{T}}$ is the displacement vector of a point belonging to a beam section; $\varepsilon=\left\{\varepsilon_{x}, \gamma_{x y}^{m}, \gamma_{x z}^{m}\right\}^{\mathrm{T}}$ is the strain vector of a point belonging to a beam section; $\mathbf{u}_{o}$ is the displacement vector of the $3 \mathrm{D}$ beam finite element corresponding to the central axis of the cross-section; $\hat{\varepsilon}$ is the generalized strain vector

$\dagger \gamma_{x y}(y, z)=\tau_{x y}(y, z) / G=\left(Q_{y} / G\right)\left(S_{z}(y) / J_{z} b(y)\right)$ and $\gamma_{y z}(y, z)=\tau_{x z}(y, z) / G=\left(Q_{z} / G\right)\left(S_{y}(z) / J_{y} b(z)\right)$, where the sectional shear force can be expressed according to Timoshenko's theory $Q_{y}=G A_{y}^{*} \gamma_{x y}^{m}, Q_{z}=G A_{z}^{*} \gamma_{x z}^{m}, G$ being the shear modulus. 

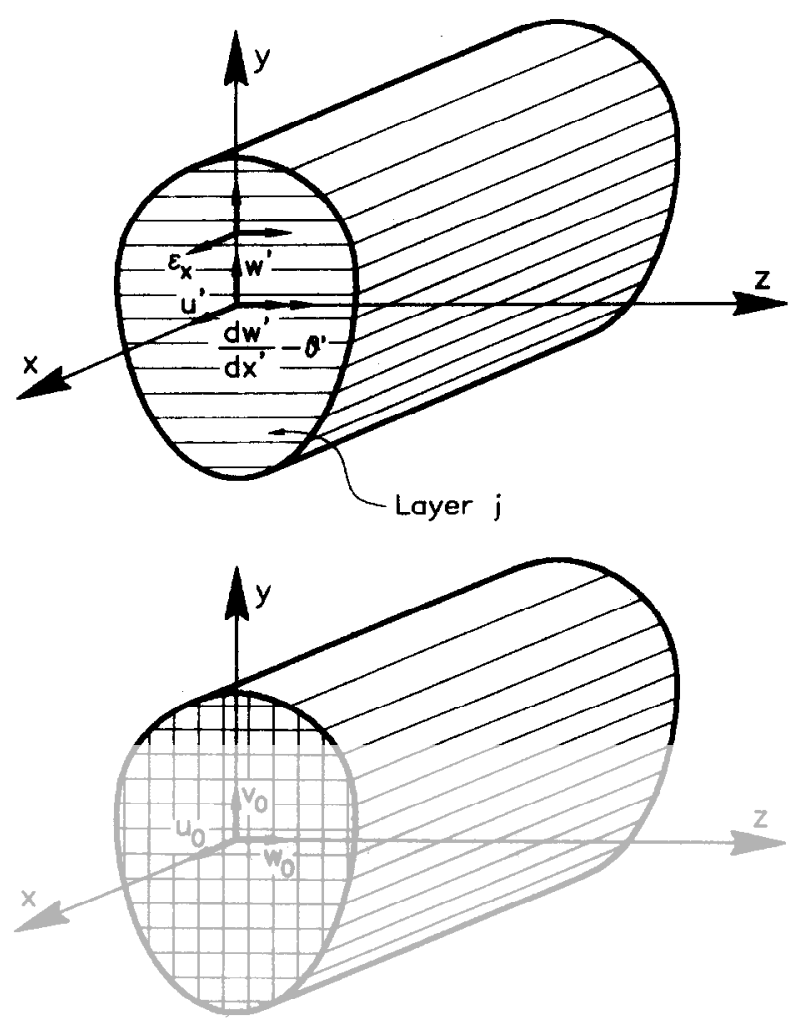

Fig. 1. (a) Layered 2D Timoshenko beam element. (b) 3D Timoshenko beam element discretized

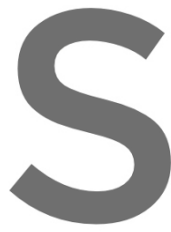
corresponding to the central axis of the beam; S is the geometric transformation matrix
relating cross-sectional variables with the point variables.
The well-known equilibrium equations are written using the virtual world principle.

The internal virtual work $L_{\text {int }}$ corresponding to a virtual strain $\delta \varepsilon$ is expressed as

Register for free at https//www.scipedia.com to download the version without the watermark

$$
\begin{aligned}
L_{i n t} & =\int_{r} \delta \varepsilon^{\mathrm{T}} \sigma_{t o t} \mathrm{~d} V=\int_{\mathscr{r}}\left(\delta \hat{\varepsilon}^{\mathrm{T}} \mathrm{S}^{\mathrm{T}}\right) \sigma_{t o t} \mathrm{~d} V \\
& =\int_{0}^{\ell} \delta \hat{\boldsymbol{\varepsilon}}^{\mathrm{T}}\left[\int_{\mathscr{\alpha}} \mathbf{S}^{\mathrm{T}} \boldsymbol{\sigma}_{t o t} \mathrm{~d} A\right] \mathrm{d} \boldsymbol{x}=\int_{0}^{\ell} \delta \hat{\boldsymbol{\varepsilon}}^{\mathrm{T}} \hat{\boldsymbol{\sigma}}_{t o t} \mathrm{~d} \boldsymbol{x}
\end{aligned}
$$

where $\mathscr{V}$ is the volume, $\mathscr{A}$ the surface of the cross-section and $\ell$ the length of the beam element, $\sigma_{t o t}=\left\{\sigma_{x}, \tau_{x y}, \tau_{x z}\right\}^{\mathrm{T}}$ are the total stresses at the point level, which are defined in detail later. The total sectional forces $\hat{\boldsymbol{\sigma}}_{t o t}=\left\{N_{x}, Q_{y}, Q_{z}, T_{x}, M_{y}, M_{z}\right\}^{\mathrm{T}}$ have been also introduced in the previous equation as

$$
\hat{\boldsymbol{\sigma}}_{t o t}=\int_{\mathscr{\infty}} \mathbf{S}^{\mathrm{T}} \boldsymbol{\sigma}_{t o t} \mathrm{~d} A
$$

A sectional density matrix $\hat{\rho}$ can be defined, relating the sectional inertia forces with the acceleration vector $\ddot{u}_{o}$ which is calculated by deriving twice equation (1) with respect to time

$$
\hat{\boldsymbol{\rho}}=\int_{\mathscr{A}} \mathbf{S}^{\mathrm{T}} \boldsymbol{\rho}_{0} \mathbf{S} \mathrm{d} A
$$

where $\rho_{0}$ is the material density. Equation (5) can be integrated for any distribution of material properties over the beam cross-section. 
Following standard finite element procedures, the discrete vector of the internal forces $\mathbf{F}_{i n t}$ and inertial forces $\mathbf{F}_{i}$ are obtained as

$$
\begin{gathered}
\mathbf{F}_{i n t}=\int_{t} \mathbf{B}^{\mathrm{T}} \hat{\boldsymbol{\sigma}}_{t o t} \mathrm{~d} x \\
\mathbf{F}_{i}=\int_{t} \mathbf{N}^{\mathrm{T}} \hat{\boldsymbol{\rho}} \ddot{\mathbf{u}}_{o} \mathrm{~d} x=\left(\int_{t} \mathbf{N}^{\mathrm{T}} \hat{\boldsymbol{\rho}} \mathbf{N} \mathrm{d} x\right) \ddot{\mathbf{a}}=\mathbf{M a ̈}
\end{gathered}
$$

where $\mathbf{N}$ and $\mathbf{B}$ are the shape function and strain matrices, $\mathbf{a}$ is the vector of nodal displacements and $\mathbf{M}$ is the elemental mass matrix. The internal forces $\mathbf{F}_{\text {int }}$ introduced in eqn (6) will be analyzed in detail in Section 4, after describing the damage model. Using now the expressions of the inertia and internal forces, the equation of motion is formulated as

$$
\mathbf{M a ̈}(t)+\mathbf{F}_{i n t}(t)=\mathbf{F}(t)
$$

\section{where $\mathbf{F}(t)$ is the vector of the dynamic load.}

As stated before, the cross-section of the beam is discretized using an orthogonal grid. Each rectangle of the grid may have different size and different materials, in this case
concrete or steel. For the concreteg visco-damage model and for the steel a simple Von Mises elasto-plastic model are used. The rectangles are defined by their comers and it is assumed that all the stresses have a linear variation over each cell of the grid. This implie solving a system of four equations with three unknowns, defining the equation of the plane which approximate by minimum squares the variation of ach component of the stes tensor. The same grid can be used to calculate all the other characteristics of the cross-

\section{VISCOUS DAMAGE CONSTITUTIVE MODEL FOR THE CONCRETE}

\subsection{General concepts}

The solution of beam structures subjected to seismic actions beyond the linear behaviour has been usually treated using: (a) theories based on plastic hinge formation (Massonet and Save, 1966). This approach has the drawback of admitting that the damage of a structure point is dominated by bending criteria, which is true only for some very particular structures. (b) Simulation of beam structures based on the concept of plastification bending moment. This procedure is based on formulating simplified curvature-bending moment constitutive laws (Clough et al., 1965, Aoyama and Sugano 1968).

The last formulations started from representing the behaviour of materials in an approximate form based mainly on experimental studies. Today, it is required that these formulations be thermodynamically sustainable. Between those which meet this latter requirement, the so-called continuous damage theory is generally accepted as an alternative in the most complex constitutive formulations (DiPasquale and Cakmak, 1989, Oliver et al., 1990). An application of this model to the dynamic case can be seen in Mazars (1991) where a column discretized in plane finite elements, subjected to seismic action, is calculated. The damage models have a rigorous but relatively simple formulation strictly based on thermodynamics (Simó and Ju, 1987). They deal with the non-linear behaviour by means of one or more internal variables called damage variables which indicate the loss of secant stiffness of the material and are normalized to a unit value which corresponds to maximum 


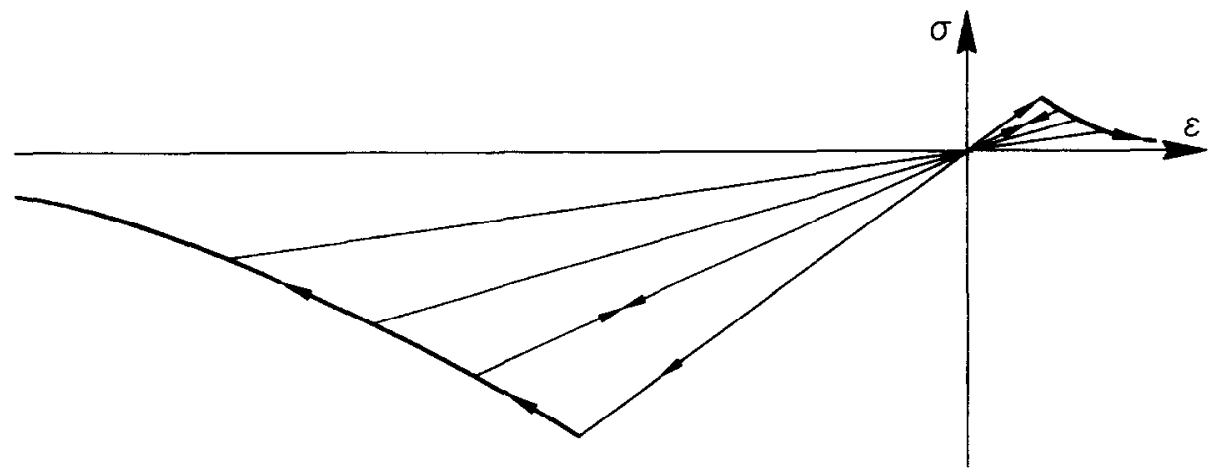

Fig. 2. Local damage behaviour.

damage. Figure 2 shows a simplified unidimensional representation of the behaviour of a point within a damaged material (Oliver et al., 1990).

The model presented herein is a $3 \mathrm{D}$ damage constitutive model based on solid mechanics and it has a single internal variable (Oliver et al., 1990). Therefore, this is a local isotropic damage model and it is based on Kachanov's theory (1958), appropriate for simulating the behaviour of concrete under monotonically increasing loads. Many ideas inherent to the model have been taken from the works of Simó and Ju (1987), Lubliner et al. (1989) and Oliver et al. (1990). This formulation has been chosen because it is a compromise between the complexity of the models describing the behaviour of the concrete and the versatility needed when dealing with dynamic problems. This insures accurate results and low cost solutions for the non-linear problems which are the object of this

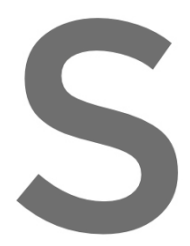
paper.

The numerical treatment of viscoelastic phenomena in materials can be followed in detail in Lubliner (1990) and Simó and Hughes (1995). The damping effect of the bean structure was simulated in this paper by using a model consisting of a damper placed in
parallel with the structure (Barbat et al., 1993; Luccion et al., 1995).

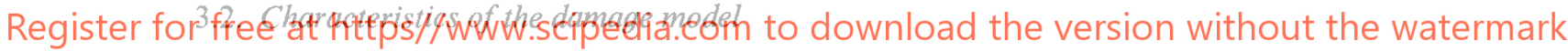

Free energy and constitutive law. The model is formulated in the material configuration, for thermodynamically stable problems, with no temperature time variation. For this specific case the following mathematical form for the free energy is assumed, where the non-damaged elastic part is expressed as a scalar quadratic function of tensorial arguments (Malvern 1969; Simó and Ju, 1987; Oliver et al., 1990)

$$
\Psi(\varepsilon ; d)=(1-d) \Psi_{o}(\varepsilon)=(1-d)\left(\frac{1}{2 \rho_{o}} \boldsymbol{c}^{\mathrm{T}} \boldsymbol{\sigma}^{o}\right)=(1-d)\left(\frac{1}{2 \rho_{o}} \boldsymbol{\varepsilon}^{\mathrm{T}} \mathbf{C}^{o} \varepsilon\right)
$$

In (9) the strain tensor $\varepsilon$ is the free variable of the problem, $d(0 \leqslant d \leqslant 1)$ is the internal damage variable, $\rho_{o}$ is the density in the material configuration and $\mathbf{C}^{o}$ is the stiffness tensor of the material in the initial undamaged state.

For stable thermical state problems the Clasius Planck dissipation inequality is valid, whose local lagrangian form is (Malvern, 1969; Lubliner, 1990)

$$
\begin{gathered}
\dot{\Xi}_{m}={ }_{\rho_{o}}^{1} \boldsymbol{\sigma}^{\mathrm{T}} \dot{\varepsilon}-\dot{\Psi} \geqslant 0 \\
\dot{\Xi}_{m}=\left(\frac{1}{\rho_{o}} \boldsymbol{\sigma}^{\mathrm{T}}-\frac{\partial \Psi}{\partial \varepsilon}\right) \dot{\varepsilon}-\frac{\partial \Psi}{\partial d} \dot{d} \geqslant 0 .
\end{gathered}
$$

This expression for the dissipation rate $\dot{\Xi}_{m}$ allows the following two considerations : 


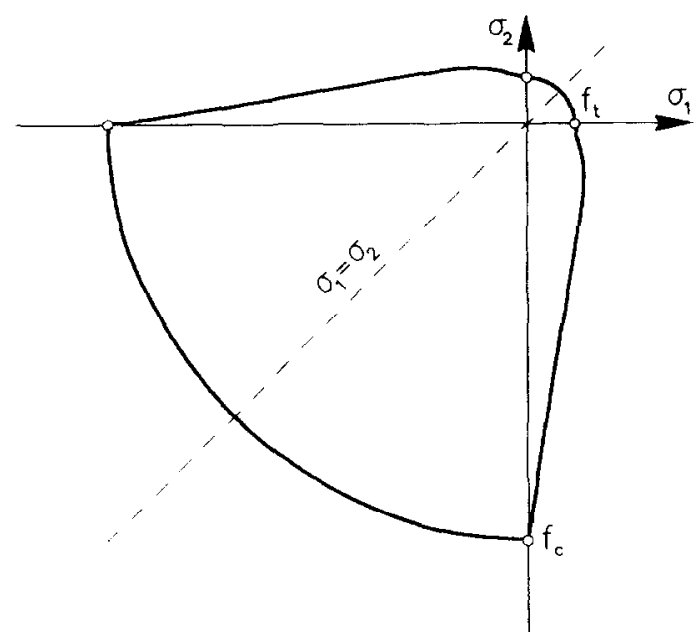

Fig. 3. Damage yield function in the principal plane $\sigma_{1}-\sigma_{2}$

(a) In order to guarantee the unconditional fulfilment of the Clasius Planck inequality (Lubliner, 1990), the multiplier of $\dot{\varepsilon}$ which represents an arbitrary temporal variation of the free variable, must be null. This condition provides the constitutive law of the damage problem :

$$
\frac{1}{\rho_{o}} \sigma^{\mathrm{T}}-\frac{\partial \Psi}{\partial \varepsilon}=0 \Rightarrow \sigma=\rho_{0}\left\{\frac{\partial \Psi}{\partial \varepsilon}\right\}^{\mathrm{T}}=(1-d) \mathbf{C}^{0} \varepsilon=\mathbf{C}^{s} \varepsilon
$$
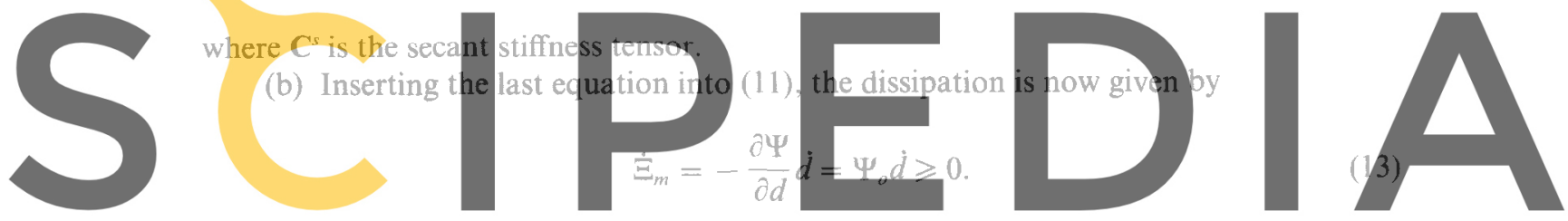

As $\Psi_{0}$ is always positive, eqn (13) states that the damage rate $d$ cannot be negative, i.e., the

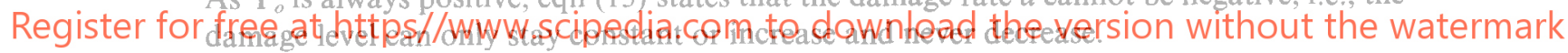

Damage yield criterion. The damage yield criterion is defined as a function of the free energy of the undamaged material, expressed in terms of the undamaged principal stresses $\sigma_{i}^{o}$, as

$$
F=K\left(\sigma^{o}\right) \sqrt{2 \rho_{o} \Psi_{o}}-1=\frac{K\left(\sigma^{o}\right)}{\sqrt{E^{o}}} \sqrt{\sum_{i=1}^{3}\left(\sigma_{i}^{o}\right)^{2}}-1 \leqslant 0
$$

where the terms of the above equation have the following meaning:

$$
\begin{gathered}
K\left(\sigma^{o}\right)=\frac{r}{\sqrt{2 \rho_{o}\left(\Psi_{t}^{o}\right)_{L}}}+\frac{1-r}{\sqrt{2 \rho_{o}\left(\Psi_{c}^{o}\right)_{L}}} ; \quad r=\frac{\sum_{i=1}^{3}\left\langle\sigma_{i}^{o}\right\rangle}{\sum_{i=1}^{3}\left|\sigma_{i}^{o}\right|} \\
2 \rho_{o}\left(\Psi_{t, c}^{o}\right)_{L}=\sum_{i=1}^{3}\left\langle \pm \sigma_{i}^{o}\right\rangle \varepsilon_{i} ; \quad\left(\Psi_{o}\right)_{L}=\left(\Psi_{t}^{o}\right)_{L}+\left(\Psi_{c}^{o}\right)_{L} .
\end{gathered}
$$

In these equations $\left(\Psi_{t, c}^{o}\right)_{L}$ represent the part of the free energy developed when the traction/compression limit is reached and $\langle \pm x\rangle=\frac{1}{2}(|x| \pm x)$ is the McAuley's function. Taking into account that the traction/compression strength are $f_{t}=\left(\Psi_{t}^{o} E^{o}\right)_{L}^{1 / 2}$ and $f_{c}=\left(\Psi_{c}^{0} E^{0}\right)_{L}^{1 / 2}$, respectively. Substituting the last definition in eqn (14), it results the damage yield function, which can be written, according to Fig. 3, as 


$$
F=\bar{\sigma}-f_{c} \leqslant 0
$$

where

$$
\bar{\sigma}=[n r+(1-r)] \sqrt{\sum_{i=1}^{3}\left(\sigma_{i}^{o}\right)^{2}}
$$

with $n=f_{c} / f_{t}$. This damage yield function, expressed in the non-damaged principal stresses space, allows a great number of choices. The advantage of the yield criterium written in eqn (15) is that any yield function $F$ can be used always as long as it is homogenous and of first order in stresses (i.e., Mohr-Coulomb, Drucker-Prager, Lubliner et al. (1989), etc.), in substitution of the equivalent stress $\partial$.

This opens the possibility of applying more accurate and powerful theories within the theoretical framework given by eqn (15). Nevertheless, the simple form provided by eqn (16) fulfils the above requirements; besides, it is simple and yields satisfactory results within the range of assumptions made for this model and therefore will be used henceforward as the scalar expression defining $\bar{\sigma}$ (Oliver et al., 1990). An expression entirely equivalent to (15), proposed by Simó (1987) with the aim of simplifying the mathematical deduction of the damage variable of the model, is the following:

$$
\bar{F}=G(\bar{\sigma})-G\left(f_{c}\right) \leqslant 0
$$

where $G(\chi)$ is a scalar monotonic function to be determined. Its shape will be chosen conveniently for the subsequent development of the damage model.
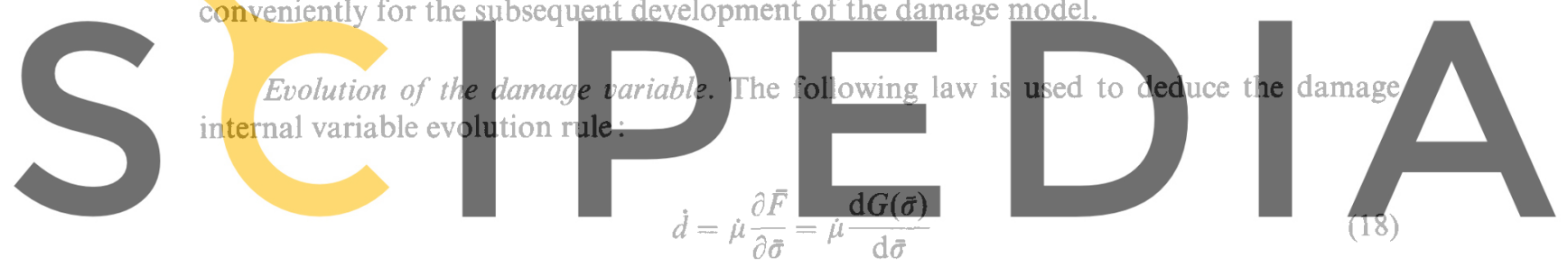

Register for free at https//www.scipedia.com to download the version without the watermark where $\mu$ is a non-negative scalar denominated damage consistency parameter, analogous to the plastic consistency parameter $\lambda$ in standard plasticity theory.

Similarly to plasticity, a yielding rule $\bar{F}=0$ and a consistency rule $\dot{F}=0$ for a point subjected to a damaging process are defined. The yielding rule and the properties of $G(\chi)$ allow to write $G(\bar{\sigma})-G\left(f_{c}\right)=0$, what implies $\bar{\sigma}=f_{c}$ and consequently

$$
\frac{\mathrm{d} G(\bar{\sigma})}{\mathrm{d} \tilde{\sigma}}=\frac{\mathrm{d} G\left(f_{c}\right)}{\mathrm{d} f_{c}}
$$

From the condition of consistency - that means persistency on the damage yield surfaceand from the properties of function $G(\chi)$, the following equation is deduced :

$$
\frac{\partial \bar{F}}{\partial \bar{\sigma}} \dot{\sigma}+\frac{\partial \bar{F}}{\partial f_{c}} \dot{f}_{c}=\frac{\mathrm{d} G(\bar{\sigma})}{\mathrm{d} \bar{\sigma}} \dot{\bar{\sigma}}-\frac{\mathrm{d} G\left(f_{c}\right)}{\mathrm{d} f_{c}} \dot{f}_{c}=0
$$

and the use of (19) allows to write $\dot{\bar{\sigma}}=\dot{f}_{c}$. Equation (20) can be now rewritten and leads to

$$
\begin{aligned}
\frac{\mathrm{d} G(\bar{\sigma})}{\mathrm{d} \tilde{\sigma}} \dot{\bar{\sigma}}=\frac{\mathrm{d} G\left(f_{c}\right)}{\mathrm{d} f_{c}} \dot{f}_{c} & =\frac{\mathrm{d} G\left(f_{c}\right)}{\mathrm{d} f_{c}} \frac{\mathrm{d} f_{c}}{\mathrm{~d}(d)} \dot{d}=\frac{\mathrm{d} G\left(f_{c}\right)}{\mathrm{d}(d)} \dot{\mu} \frac{\mathrm{d} \bar{G}(\bar{\sigma})}{\mathrm{d} \bar{\sigma}} \\
\dot{\sigma} & =\frac{\mathrm{d} G\left(f_{c}\right)}{\mathrm{d}(d)} \dot{\mu} .
\end{aligned}
$$




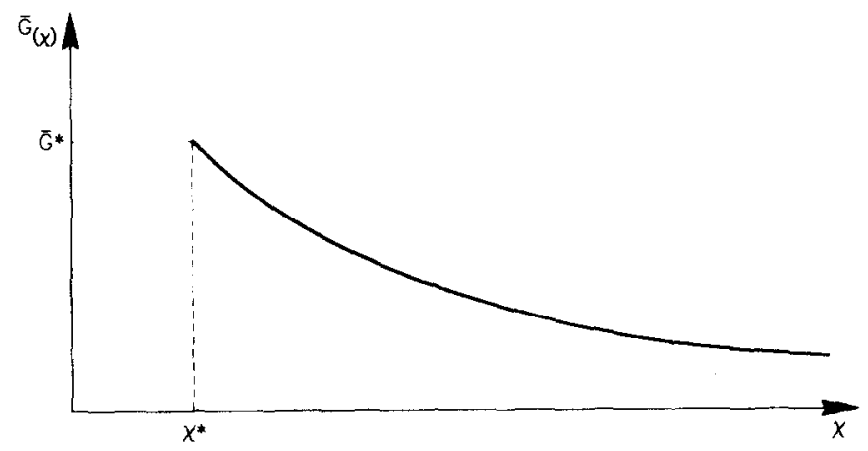

Fig. 4. Representation of the chosen $G(\chi)$.

Conveniently choosing $G\left(f_{c}\right)$ as the function which describes the evolution of the damage $\left[d=G\left(f_{c}\right)\right]$, the damage consistency parameter $\dot{\mu}$ can be expressed as

$$
\dot{\mu}=\dot{\bar{\sigma}}=\dot{\bar{\omega}}=\frac{\partial \bar{\sigma}}{\partial \sigma^{\circ}} \dot{\sigma}^{\circ}=\frac{\partial \bar{\sigma}}{\partial \sigma^{\circ}} \mathbb{C}^{0} \dot{\varepsilon} .
$$

Substituting this equation into (18) and (14), the following expressions which formulate the temporal evolution of the damage and dissipation variables are obtained:
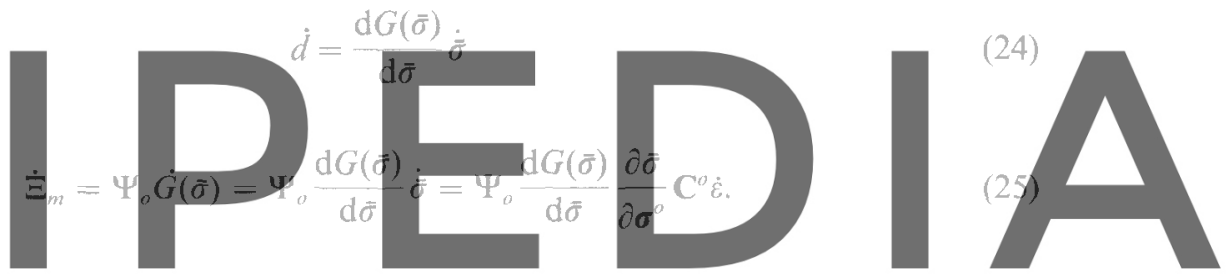

The loading/unloading condition is derivated from the relations of Kuhn-Tucker for-

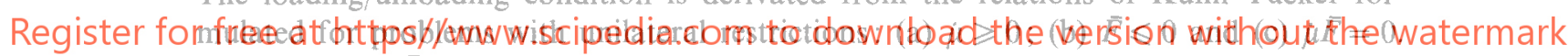
From these, if $\bar{F}<0$, then the third condition imposes $\mu=0$ and, if $\dot{\mu}>0$, then the same condition requires that $F=0$.

Definition of function $\mathrm{G}$. From the different alternatives for defining function $G(\chi)$ (Simó and Ju, 1987), the following equation was chosen

$$
G(\chi)=1-\frac{\bar{G}(\chi)}{\chi}
$$

where $\bar{G}(\chi)$ describes a function so that it gives for $\chi=\chi^{*}$ the compression initial yield tension $\bar{G}^{*}$ and for $\chi \rightarrow \infty$ the final strength $\bar{G} \rightarrow 0$. Thus, by running all the deformation path, the point will have dissipated an energy equivalent to the specific fracture energy. In our work, the exponential function proposed by Oliver et al. (1990), which is shown in Fig. 4 , was used

$$
\bar{G}(\chi)=\chi^{*} e^{A\left[1-\left(x / x^{*}\right)\right]} ; \quad G(\chi)=1-\frac{\chi^{*}}{\chi} e^{A\left[1-\left(\chi / x^{*}\right)\right]}
$$

For a uniaxial traction process under monotonically increasing load, the temporal dissipation change is given by (14), with $\sigma=n \sigma_{t}$ and $\bar{\Psi}_{o}=\frac{1}{2} \varepsilon_{t} E^{o} \varepsilon_{t}=\left(\sigma_{t}\right)^{2} / 2 E^{o}=\bar{\sigma}^{2} / 2 n^{2} E^{o}$. Integrating (14) in time we can calculate the total dissipated energy at the end of the uniaxial traction process as 


$$
\Xi_{t}^{\max }=\int_{\sigma^{*}}^{\infty} \frac{\bar{\sigma}^{2}}{2 \rho_{o} n^{2} E^{o}} \frac{\mathrm{d} G(\bar{\sigma})}{\mathrm{d} \bar{\sigma}} \mathrm{d} \bar{\sigma}=\int_{\sigma^{*}}^{\infty} \frac{\bar{\sigma}^{2}}{2 \rho_{o} n^{2} E^{o}} \mathrm{~d} G(\bar{\sigma})
$$

and after operating we get

$$
\Xi_{t}^{\max }=\frac{\left(\bar{\sigma}^{*}\right)^{2}}{\rho_{o} n^{2} E^{o}}\left[\frac{1}{2}+\frac{1}{A}\right]
$$

giving

$$
A=\frac{1}{\frac{\Xi_{t}^{\max } \rho_{o} n^{2} E^{o}}{\left(\bar{\sigma}^{*}\right)^{2}}-\frac{1}{2}}
$$

where $\bar{\sigma}^{*}$ is the initial damage stress. Parameter $A$ is never negative, as the material must dissipate at least the energy accumulated when reaching the initial damage stress $\sigma^{*}$. Making the same hypotheses for a uniaxial compression process and postulating that parameter $A$ must be the same in both cases, it is deduced that
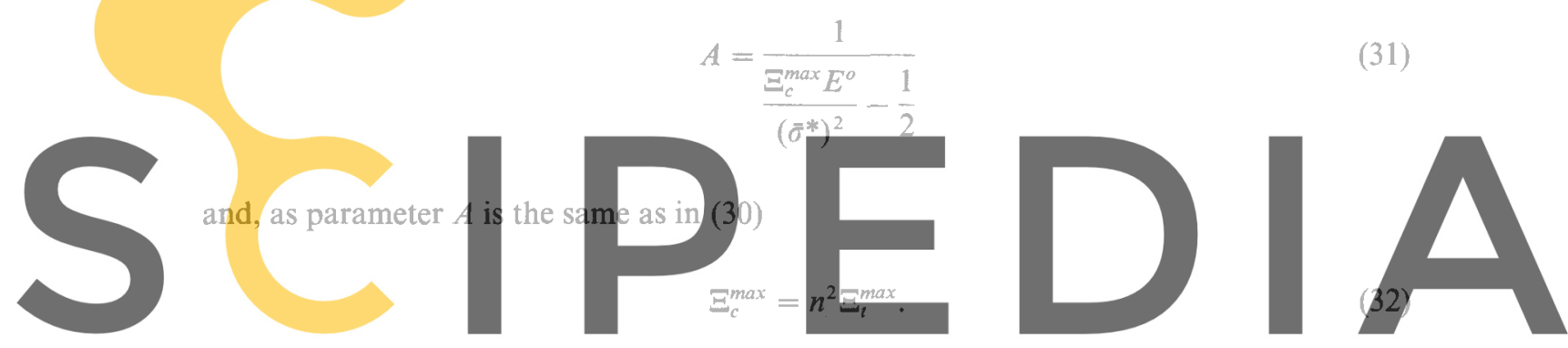

The value of traction maximum dissipation $\Xi_{t}^{\max }$ is an input of the problem and is equal to

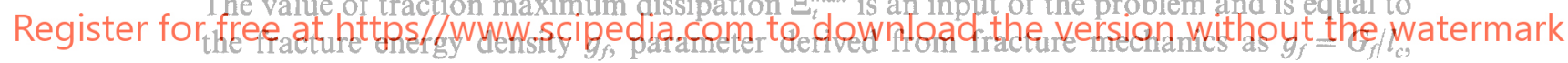

where $G_{f}$ is the fracture energy and $l_{c}$ is the characteristic length of the fractured domain (Lubiiner et ai., 1989).

Tangent constitutive law. From (12), the variation of the stress tensor and finally the unsymmetric tangent constitutive tensor $\mathbf{C}^{D}$ of the damage model can bc deduced as (Barbat et al., 1993)

$$
\begin{gathered}
\delta \boldsymbol{\sigma}=\mathbf{C}^{s} \delta \varepsilon+\delta \mathbf{C}^{s} \boldsymbol{\varepsilon} ; \quad \delta \mathbf{C}^{s}=\frac{\partial \mathbf{C}^{s}}{\partial \boldsymbol{d}} \delta d=-\mathbf{C}^{o} \delta d \\
\delta \boldsymbol{\sigma}=\mathbf{C}^{D} \delta \boldsymbol{\varepsilon}=\left[(1-d) \mathbf{I}-\frac{\mathrm{d} G(\boldsymbol{\sigma})}{\mathrm{d} \bar{\sigma}} \boldsymbol{\sigma}^{o} \otimes \frac{\partial \bar{\sigma}}{\partial \boldsymbol{\sigma}^{o}}\right] \mathbf{C}^{o} \delta \boldsymbol{\varepsilon}=(\mathbf{I}-\mathbf{D}) \mathbf{C}^{o} \delta \boldsymbol{\varepsilon}
\end{gathered}
$$

where

$$
\mathbf{C}^{D}=(\mathbf{I}-\mathbf{D}) \mathbf{C}^{o}
$$

In these equations, $\mathbf{I}$ is the identity matrix of the same order as $\mathbf{C}^{\circ}$ and $\mathbf{D}$ is a non-symmetric matrix, depending only on the stress vector $\sigma^{\circ}$ in the undamaged material, as the damage variable is also implicitly related with the mentioned stress vector through the equivalent stress $\bar{\sigma}$. 


\subsection{Visco-elastic effects}

The effect of damping on each compounding material of the beam structure is now considered by means of a Kelvin model (Malvern 1969; Luccioni et al., 1995). Each point of the material undergoes the same deformation $\varepsilon$, so that the total stress $\sigma_{t o t}$ of the system will be the sum of a non-viscous stress $\sigma$ and a viscous stress $\sigma_{\text {vis }}$, i.e.

$$
\sigma_{t o t}=\sigma+\sigma_{v i s}=\mathbf{C}^{s} \boldsymbol{\varepsilon}+\boldsymbol{\eta}^{s} \dot{\varepsilon}
$$

where the secant viscous constitutive matrix $\eta^{s}$ is defined here as

$$
\boldsymbol{\eta}^{s}=\frac{\eta}{E^{o}} \mathbf{C}^{s}=\alpha \mathbf{C}^{s}
$$

The viscous tensor definition made in this equation is based on the hypothesis that at the end of the load process a material point remains completely relaxed, without stiffness nor cohesion between particles. This leads to the dissipation of the entire energy of the material point, thus the material remaining unable to withstand any load. For this reason, it is assumed in this work that the material point does not preserve its initial viscous characteristics. However, this hypothesis is flexible and can be adapted to the material type without affecting the subsequent general formulation. In eqn (36), $\eta$ is the one-dimensional viscous parameter and $\alpha$ is the relaxation time, defined as the time needed by the elasto-viscous system to reach a stable configuration in the undamaged state.

With this assumption, the behaviour of the system under virtual variations in strains
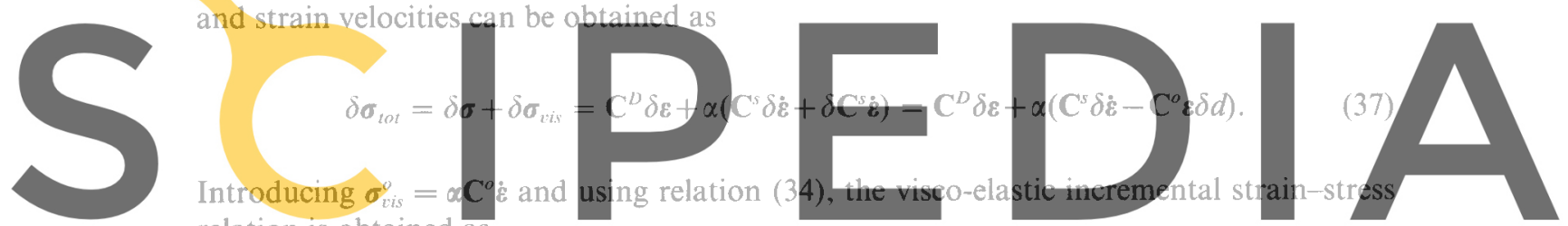

relation is obtained as

Register for free at https//www.scipedia.com to download the version without the watermark

where $\mathbf{D}_{v t s}$ takes the following value (Barbat et al., 1993):

$$
\mathbf{D}_{v i s}=d \mathbf{I}+\frac{\mathrm{d} G(\tilde{\sigma})}{\mathrm{d} \bar{\sigma}}\left(\boldsymbol{\sigma}^{o}+\boldsymbol{\sigma}_{v i s}^{o}\right) \otimes \frac{\partial \bar{\sigma}}{\partial \boldsymbol{\sigma}^{o}}
$$

\section{ELASTO-PLASTIC MODEL FOR THE STEEL BARS}

As it has been shown before, the steel bars have been represented by two- or threedimensional steel layers or fibers. The behaviour of these steel layers or fibers is simulated by means of an elasto-perfectly plastic constitutive model, with a Von Mises yield surface and associated plasticity. Due to the fact that this model is well known, reference is made to the classical works of Malvern (1969) and Lubliner (1990).

\section{APPLICATION OF THE VISCO-DAMAGE CONSTITUTIVE LAW TO THE STRUCTURAL MODEL}

The secant and tangent form of the visco-damage constitutive model are deduced in this section. The first one is required because it is used in the integration of the mentioned constitutive model; the second one allows to deduce the constitutive tensor needed in establishing the tangent stiffness and damping matrices. 


\subsection{Secant equilibrium law}

Considering eqn (35), the sectional forces-eqn (4)—can be expressed as

$$
\hat{\sigma}_{t o t}=\int_{\infty} \mathbf{S}^{\mathrm{T}} \sigma_{t o t} \mathrm{~d} A=\int_{\mathscr{\infty}} \mathbf{S}^{\mathrm{T}} \mathbf{C}^{s} \varepsilon \mathrm{d} A+\int_{\infty} \mathbf{S}^{\mathrm{T}} \eta^{s} \dot{\varepsilon} \mathrm{d} A
$$

where

$$
\boldsymbol{\sigma}=\int_{\mathscr{A}} \mathbf{S}^{\mathrm{T}} \mathbf{C}^{s} \boldsymbol{\mathrm { d }} A, \quad \boldsymbol{\sigma}_{v t s}=\int_{\mathscr{\infty}} \mathbf{S}^{\mathrm{T}} \boldsymbol{\eta}^{s} \dot{\mathrm{g}} \mathrm{d} A
$$

Substituting now $\varepsilon$ from eqn (2) and its derivative in (40), the sectional forces become

$$
\hat{\boldsymbol{\sigma}}_{t o t}=\left(\int_{\mathscr{A}} \mathbf{S}^{\mathrm{T}} \mathbf{C}^{s} \mathbf{S} \mathrm{d} A\right) \hat{\boldsymbol{\varepsilon}}+\left(\int_{\mathscr{A}} \mathbf{S}^{\mathrm{T}} \eta^{s} \mathbf{S} \mathrm{d} A\right) \dot{\hat{\varepsilon}}
$$

This equation can be written in the following compact form

$$
\hat{\boldsymbol{\sigma}}_{t o t}=\hat{\mathbf{C}}^{s} \hat{\boldsymbol{\varepsilon}}+\hat{\boldsymbol{\eta}}^{s} \dot{\hat{\boldsymbol{\varepsilon}}}
$$

where the definitions

$$
\hat{\mathbf{C}}^{s}=\int_{\mathscr{A}} \mathbf{S}^{\mathrm{T}} \mathbf{C}^{s} \mathbf{S} \mathrm{d} A, \quad \hat{\boldsymbol{\eta}}^{s}=\int_{\mathscr{A}} \mathbf{S}^{\mathrm{T}} \boldsymbol{\eta}^{s} \mathbf{S} \mathrm{d} A
$$

have been used. According to standard finite element analysis (see also Section 2), the derivative of the generalized strain vector is $\dot{\hat{\mathbf{E}}}=\mathbf{B} \dot{\mathbf{a}} ;$ in this case, the sectional forces (eqn 43) can be written in the following form:

$$
\hat{\boldsymbol{\sigma}}_{\text {tot }}=\hat{\mathbf{C}}^{s} \mathbf{B a}+\hat{\boldsymbol{\eta}}^{s} \mathbf{B} \mathbf{a}
$$

Finally, the vector of the internal forces-eqn (6)-can be rewritten as

$$
\mathbf{F}_{i n t}=\int_{\ell} \mathbf{B}^{\mathrm{T}} \hat{\boldsymbol{\sigma}}_{t o t} \mathrm{~d} x=\left(\int_{\ell} \mathbf{B}^{\mathrm{T}} \hat{\mathbf{C}}^{s} \mathbf{B} \mathrm{d} x\right) \mathbf{a}+\left(\int_{\ell} \mathbf{B}^{\mathrm{T}} \hat{\eta}^{s} \mathbf{B} \mathrm{d} x\right) \dot{\mathbf{a}} .
$$

Introducing the notations

$$
\mathbf{K}_{S E C}=\int_{\ell} \mathbf{B}^{\mathrm{T}} \hat{\mathbf{C}}^{s} \mathbf{B} \mathrm{d} x, \quad \mathbf{D}_{S E C}=\int_{\ell} \mathbf{B}^{\mathrm{T}} \hat{\boldsymbol{\eta}}^{s} \mathbf{B} \mathrm{d} x
$$

eqn (45) is rewritten as

$$
\mathbf{F}_{i n t}=\mathbf{K}_{S E C} \mathbf{a}+\mathbf{D}_{S E C} \dot{\mathbf{a}} .
$$

\subsection{Tangent equilibrium law}

The variation of the sectional forces can be expressed starting from (4) in the following form:

$$
\delta \hat{\boldsymbol{\sigma}}_{t o t}=\int_{\mathscr{A}} \mathbf{S}^{\mathrm{T}} \delta \boldsymbol{\sigma}_{t o t} \mathrm{~d} \boldsymbol{A}
$$

Writing now the variation of the total stresses $\sigma$ of the system using the eqns (37) and (38) 


$$
\delta \sigma_{i o t}=\delta \sigma_{v i s}=\left(\mathbf{I}-\mathbf{D}_{v i s}\right) \mathbf{C}^{o} \delta \varepsilon+\alpha \mathbf{C}^{s} \delta \dot{\varepsilon}
$$

eqn (47) becomes

$$
\delta \hat{\boldsymbol{\sigma}}_{t o t}=\int_{\phi} \mathbf{S}^{\mathrm{T}}\left[\left(\mathbf{I}-\mathbf{D}_{v i s}\right) \mathbf{C}^{o}\right] \delta \boldsymbol{\varepsilon} \mathrm{d} A+\int_{s} \mathbf{S}^{\mathrm{T}} \alpha \mathbf{C}^{s} \delta \dot{\varepsilon} \mathrm{d} A
$$

Substituting $\varepsilon$ given by (2) and its derivative in (49), the variation of the section forces takes the form

$$
\delta \hat{\boldsymbol{\sigma}}_{t o t}=\left[\int_{\mathscr{\sigma}} \mathbf{S}^{\mathrm{I}}\left(\mathbf{I}-\mathbf{D}_{v i s}\right) \mathbf{C}^{o} \mathbf{S d} A\right] \delta \hat{\boldsymbol{\varepsilon}}+\left[\int_{\mathscr{A}} \mathbf{S}^{1} \alpha \mathbf{C}^{s} \mathbf{S} \mathrm{d} A\right] \delta \hat{\hat{\varepsilon}}
$$

which, using similar developments as those used in Section 4.1., can be written as

$$
\delta \hat{\boldsymbol{\sigma}}_{u, t}=\hat{\mathbf{C}}^{D} \mathbf{B} \delta \mathbf{a}+\hat{\boldsymbol{\eta}}^{s} \mathbf{B} \delta \dot{\mathbf{a}}
$$

where

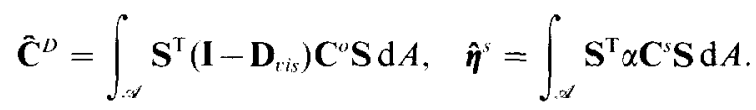

Using these equations, the variation of the internal forces vector is expressed in the following form

$$
\delta \mathbf{F}_{i n t}=\int \mathbf{B}^{\mathrm{T}} \delta \hat{\boldsymbol{\sigma}}_{t o t} \mathrm{~d} x=\left(\int \mathbf{B}^{\mathrm{T}} \overline{\mathbf{C}}^{D} \mathbf{B} \mathrm{d} x\right) \delta \mathbf{a}+\left(\int \mathbf{B}_{t}^{\mathrm{T}} \hat{\boldsymbol{\eta}}^{s} \mathbf{B} \mathrm{d} x\right) \delta \dot{\mathbf{a}}
$$

Introducing the notations

$$
\mathbf{K}_{T A N}=\int_{t} \mathbf{B}^{\mathrm{T}} \hat{\mathbf{C}}^{D} \mathbf{B} \mathrm{d} x, \quad \mathbf{D}_{T A N}=\mathbf{D}_{s e t}=\int_{t} \mathbf{B}^{\mathrm{T}} \hat{\eta}^{s} \mathbf{B} \mathrm{d} x
$$

eqn (53) is finally written as

$$
\delta \mathbf{F}_{i n t}=\mathbf{K}_{T A N} \delta \mathbf{a}+\mathbf{D}_{T A N} \delta \dot{\mathbf{a}} .
$$

\section{GLOBAL DAMAGE INDICES}

The starting point for deducing a global structural damage index is eqn (9), which relates the damaged part of the free energy $\Psi$ with the non-damaged elastic free energy $\Psi_{n}$. In order to find a global index, a similar expression is deduced by integrating (9) over the entire volume of the structure as follows:

$$
\Psi=(1-d) \Psi_{o} \Rightarrow W_{p}=\int_{\succ} \Psi \mathrm{d} V=\int_{\gamma^{\prime}}(1-d) \Psi_{o} \mathrm{~d} V=(1-D) W_{p}^{o}
$$

where $D$ is the global damage index, $W_{p}^{o}=\int_{\gamma} \Psi_{o} \mathrm{~d} V$ is the total potential energy of the structure considered as undamaged and $W_{p}$ is the total potential energy corresponding to the actual damaged state. Solving eqn (55) for $D$, the following final relation is obtained : 


$$
D=1-\frac{W_{p}}{W_{p}^{o}}=\frac{\int_{\mathscr{r}} \Psi_{o} \mathrm{~d} V-\int_{\mathscr{r}}(1-d) \Psi_{o} \mathrm{~d} V}{\int_{\mathscr{r}} \Psi_{o} \mathrm{~d} V}=\frac{\int_{\mathscr{r}} \mathrm{d} \Psi_{o} \mathrm{~d} V}{\int_{\mathscr{r}} \Psi_{o} \mathrm{~d} V}
$$

If a damage index for a part or member of the structure is needed (such as floors, columns, etc) the integration will be performed only over that specific part.

In a finite element scheme, the damage index $D_{p}$ of a beam cross-section is given by a similar expression obtained by integrating (9) over the cross-section of the beam, with $\Psi_{o}=\frac{1}{2} \varepsilon^{\mathrm{T}} \boldsymbol{\sigma}_{\text {tot }}^{o}$ and $\boldsymbol{\varepsilon}=\mathrm{S} \hat{\boldsymbol{\varepsilon}}$, i.e.

$$
D_{p}=1-\frac{\hat{\boldsymbol{\varepsilon}}^{\mathrm{T}} \hat{\boldsymbol{\sigma}}_{t o t}}{\hat{\boldsymbol{\varepsilon}}^{\mathrm{T}} \hat{\boldsymbol{\sigma}}_{t o t}^{o}} ; \quad \hat{\boldsymbol{\sigma}}_{t o t}=\int_{\mathscr{A}} \mathbf{S}^{\mathrm{T}} \boldsymbol{\sigma}_{t o t} \mathrm{~d} A=\int_{\mathscr{A}}(1-d) \mathbf{S}^{\mathrm{T}} \boldsymbol{\sigma}_{t o t}^{o} \mathrm{~d} \boldsymbol{A}
$$

where $\hat{\varepsilon}$ and $\hat{\sigma}_{t o t}$ are the generalized strains and stresses in that beam cross-section, respectively.

Substituting the value of the free energy in eqn (56), the global damage index will take the following form:

$$
D=1-\frac{\sum_{(e)} \mathbf{a}^{(e) \mathrm{T}} \int_{\ell^{(e)}} \mathbf{B}^{(e) \mathrm{T}} \hat{\boldsymbol{\sigma}}_{t o t}^{(e)} \mathrm{d} s}{\sum_{(e)} \mathbf{a}^{(e) \mathrm{T}} \int_{\ell^{(e)}} \mathbf{B}^{(e) \mathrm{T}} \hat{\boldsymbol{\sigma}}_{t o t}^{(e)} \mathrm{d} s}
$$

where the sum is performed over the beam elements for which the global damage index is calculated. This damage index is similar to that proposed by DiPasquale and Cakmak (1989).

\section{NUMERICAL IMPLEMENTATION}

The implementation process of the visco-damage model in a finite element computer program is explained in Tables 1,2 and 3. The implicit time integration scheme of Newmark for nonlinear problems is described in Table 1 (Barbat and Miquel Canet, 1989; Barbat $e t$ al., 1993). This scheme has been included in the finite element program which has been used in the present work. The sectional forces and the constitutive tensors are described in Table 2, which is called at point B-III of Table 1. Table 2 shows the decomposition of the cross-sectional displacements at a point on the beam axis (eqns 1 and 2) into $n$ strain tensors corresponding to each of the $n$ points of the cross-sectional net (see Figure 1b). Once known the strain field at each point of the cross-section, the corrected stress values are obtained integrating the visco-damage constitutive equation (see Sections 3, 4 and 5). In a general case, in which the damage is not defined through the eqn (27) and function $G(\chi)$ may not be integrable in a closed form, an incremental integration approach as described in Table 3 can be undertaken. Starting from these stresses, an inverse transformation is performed according to eqns (4) and (50) (see Table 2), which allows to "translate" the $n$ stress tensors into a single set of sectional forces corresponding to the beam axis. The values obtained for these sectional forces are then introduced in Table 1 to calculate the residual forces in order to check the convergence of the nonlinear process. Resuming, the block scheme of Table 3 is called within Table 2 for evaluating the constitutive characteristics of the model. Table 2 is called in Table 1, within the point B-III, to compute the sectional forces and the tangent and secant constitutive tensors. 
A. First iteration (passage from time instant $i$ to time instant $i+1$ )

$\triangleright$ Update relevant matrices

$$
\mathbf{K}_{S E C}=\int_{r} \mathbf{B}^{\mathrm{T}} \hat{\mathbf{C}}^{\mathrm{s}} \mathbf{B} \mathrm{d} x ; \quad \mathbf{K}_{T A N}=\int_{r} \mathbf{B}^{\mathrm{T}} \hat{\mathbf{C}}^{D} \mathbf{B} \mathrm{d} x ; \quad \mathbf{D}_{S E C}=\mathbf{D}_{T A N}=\int_{r} \mathbf{B}^{\mathrm{T}} \hat{\eta}^{s} \mathbf{B} \mathrm{d} x
$$

$\triangleright$ Compute

$$
\begin{gathered}
\hat{\mathbf{K}}=\frac{1}{\beta \Delta t^{2}} \mathbf{M}+\frac{\gamma}{\beta \Delta t} \mathbf{D}_{T A N}+\mathbf{K}_{T A N} \\
\hat{\mathbf{F}}_{i+1}^{(1)}=\mathbf{F}\left(t_{i+1}\right)+\mathbf{M}\left[\frac{1}{\beta \Delta t} \dot{\mathbf{a}}_{i}+\left(\frac{1}{2 \beta}-1\right) \ddot{\mathbf{a}}_{i}\right]-\underbrace{\left(\mathbf{D}_{T A N}-\mathbf{D}_{S E C}\right) \dot{\mathbf{a}}_{i}}_{O}-\mathbf{D}_{T A N}\left[\left(1-\frac{\gamma}{\beta}\right) \dot{\mathbf{a}}_{i}+\left(1-\frac{\gamma}{2 \beta}\right) \Delta t \ddot{\mathbf{a}}_{i}\right]-\mathbf{K}_{S E C} \mathbf{a}_{i}
\end{gathered}
$$

$\triangleright$ Calculate the first approximations for the time instant $i+1$ :

$$
\begin{aligned}
\ddot{\mathbf{a}}_{i+1}^{(1)} & -\frac{1}{\beta \Delta t^{2}} \Delta \mathbf{a}_{i+1}^{(1)}-\frac{1}{\beta \Delta t} \dot{\mathbf{a}}_{i}-\left(\frac{1}{2 \beta}-1\right) \ddot{\mathbf{a}}_{i} \\
\Delta \mathbf{a}_{i+1}^{(1)}=\hat{\mathbf{K}}^{-1} \hat{\mathbf{F}}_{i+1}^{(1)} & \dot{\mathbf{a}}_{i+1}^{(1)}=\frac{\gamma}{\beta \Delta t} \Delta \dot{\mathbf{a}}_{i+1}^{(1)}+\left(1-\frac{\gamma}{\beta}\right) \dot{\mathbf{a}}_{i}+\left(1-\frac{\gamma}{2 \beta}\right) \Delta t \ddot{\mathbf{a}}_{i} \\
\mathbf{a}_{i+1}^{(1)} & =\Delta \mathbf{a}_{i+1}^{(1)}+\mathbf{a}_{i}
\end{aligned}
$$

- B. Second and subsequent iterations (seeking the equilibrium for the time instant $i+1$ ) Loop over global convergence interactions: $j$ th iteration

$\triangleright$ I. Update relevant matrices

$$
\begin{gathered}
\mathbf{K}_{S E C}=\int_{t} \mathbf{B}^{\mathrm{T}} \hat{\mathbf{C}}^{s} \mathbf{B} \mathrm{d} x ; \quad \mathbf{K}_{T A N}=\int_{t} \mathbf{B}^{\mathrm{T}} \hat{\mathbf{C}}^{D} \mathbf{B} \mathrm{d} x ; \quad \mathbf{D}_{S E C}=\int_{t} \mathbf{B}^{\mathrm{T}} \hat{\boldsymbol{\eta}}^{s} \mathbf{B} \mathrm{d} x=\mathbf{D}_{T A N} \\
\hat{\mathbf{K}}=\frac{1}{\beta \Delta t^{2}} \mathbf{M}+\frac{\gamma}{\beta \Delta t} \mathbf{D}_{T A N}+\mathbf{K}_{T A N} \\
\hat{\mathbf{F}}_{i+1}^{(\prime+1)}=\mathbf{F}_{i n t}\left(t_{i+1}\right)-\mathbf{M} \ddot{\mathbf{a}}_{i+1}^{(1)}-\mathbf{D}_{S E C} \dot{\mathbf{a}}_{i+1}^{(j)}-\mathbf{K}_{S E C} \mathbf{a}_{i+1}^{(j)}
\end{gathered}
$$

$\triangleright$ II. If the residual forces norm $\left\|\hat{\mathbf{F}}_{i+1}^{v+1}\right\| \leqslant \varepsilon$, end of iterations and beginning of the computations in the next time step. If not, proceed calculating:

$$
\begin{aligned}
\ddot{\mathbf{a}}_{i+1}^{(j+1)} & =\frac{1}{\beta \Delta t^{2}} \delta \mathbf{a}_{i+1}^{(i+1)}+\ddot{\mathbf{a}}_{i+1}^{(j)} \\
\delta \mathbf{a}_{i+1}^{(i+1)}=\hat{\mathbf{K}}^{-1} \hat{\mathbf{F}}_{i+1}^{(j+1)} \quad & \dot{\mathbf{a}}_{i+1}^{(j+1)}=\frac{\gamma}{\beta \Delta t} \delta \mathbf{a}_{i+1}^{(j+1)}+\dot{\mathbf{a}}_{i+1}^{(j)} \\
\mathbf{a}_{i+1}^{(j+1)} & =\delta \mathbf{a}_{i+1}^{(j+1)}+\mathbf{a}_{i+1}^{(j)}
\end{aligned}
$$

III. Compute the sectional forces and the tangent and secant constitutive tensors

Cross sectional forces decomposition at each Gauss point (see Table 2)

IV. Back to step I

\section{NUMERICAL EXAMPLES}

\section{Example 1}

The simulation of the evolution of the damage process in a reinforced concrete plane frame (Fig. 5) subjected to dynamic loading is first analyzed.

The frame is $9 \mathrm{~m}$ high and $6 \mathrm{~m}$ wide and has three levels. The columns have a 30 $\mathrm{cm} \times 30 \mathrm{~cm}$ cross-section of reinforced concrete with a $4.35 \%$ steel ratio. The horizontal beams are $40 \mathrm{~cm}$ thick and $30 \mathrm{~cm}$ wide, with a steel ratio of $5.3 \%$. The structure was discretized in 45 quadratic three-noded beam finite elements having two Gauss points each. Thus, the resulting dynamic model has 87 nodes with three degrees of freedom per node. Each element is one metre long and has the cross-section divided in 20 layers of equal thickness. The 2nd and 19th layer are made of steel and the rest of concrete. The steel ratio was controlled by modifying the width of the steel layers. The state of the material is checked at the interface between layers and afterwards interpolated linearly across each layer. This gives 40 check points per cross-section in each Gauss point. The materials have 


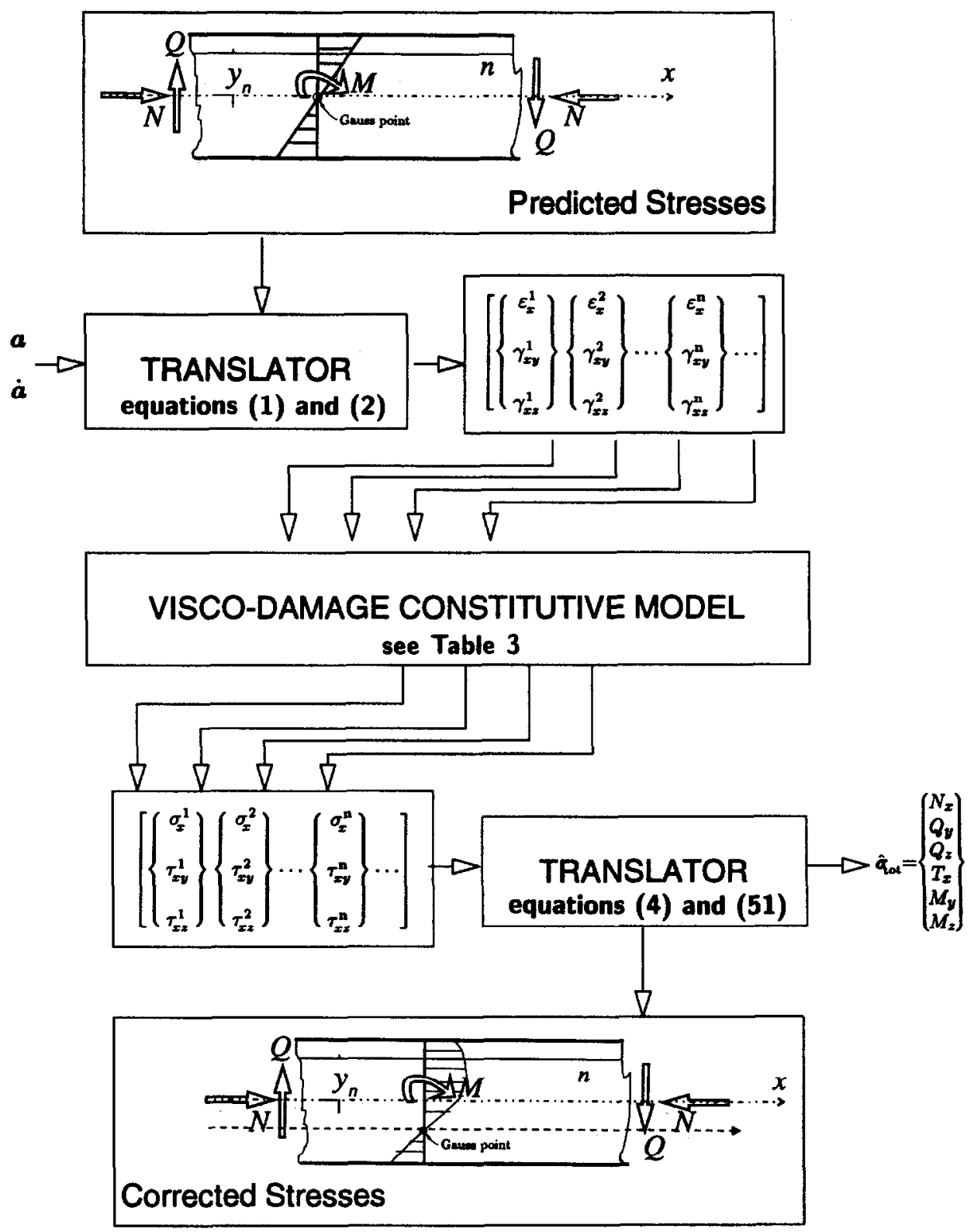

the following properties: (a) steel $E=2.1 \times 10^{6} \mathrm{daN} / \mathrm{cm}^{2}, \sigma^{\circ}=4,200 \mathrm{daN} / \mathrm{cm}^{2}, v=0.25$; $\rho_{o}=8 \mathrm{~g} / \mathrm{cm}^{3}$; (b) concrete $E=2.0 \times 10^{5} \mathrm{daN} / \mathrm{cm}^{2}, \sigma^{o}=300 \mathrm{daN} / \mathrm{cm}^{2}, v=0.17, \rho_{o}=2.5$ $\mathrm{g} / \mathrm{cm}^{3}$.

The equations of motion governing the dynamic behaviour of the structure have been solved using the Newmark algorithm with $\beta=0.25$ and $\gamma=0.5$. The initial stiffness method was chosen as nonlinear solution scheme due to the negative definition of the tangent stiffness matrix when softening effects occur. The time step used was a thirtieth of the fundamental period of the structure. As the integration of the constitutive law can be done analytically, an explicit formula [eqns (23), (26)] was used for the local damage index, thus reducing remarkably the solution cost.

The structure was calculated in two load cases: (a) subjected to a synthetic earthquake accelerogram (Fig. 6) having a predominant frequency of $4 \mathrm{~Hz}$ and a maximum amplitude 
Table 3. Visco-damage constitutive model at layer level $n$

1. Input: strains and strain velocities

$$
\Rightarrow \varepsilon_{i}^{(j)} \quad \dot{\boldsymbol{\varepsilon}}_{i}^{(j)}
$$

2. Compute the predicted non-damaged stresses for the load step $i$ and the global convergence iteration $j$

$$
\left(\boldsymbol{\sigma}^{o}\right)_{i}^{(j)}=\left(\mathbf{C}^{0}\right)_{1}^{(0)} \varepsilon_{i}^{(j)}
$$

3. General form to integrate the damage constitutive equation (Euler Backward Scheme) Loop over inner convergence iterations: $k$ th iteration

$$
\begin{aligned}
& \text { for : } k=1 \Rightarrow \sigma_{i}^{(j, v)}=\left(\sigma^{o}\right)_{i}^{(j)} \\
& \circledast \sigma_{i}^{(j, k)}=\left(1-d_{i}^{(j, k)}\right) \sigma_{i}^{(j, \omega)} \\
& \bar{\sigma}_{i}^{(j k)}=\bar{\sigma}\left(\boldsymbol{\sigma}_{i}^{(j)}\right) \\
& \text { If } F(\bar{\sigma}, d) \leqslant 0 \text { [eqn }(17)] \Rightarrow \text { no damage } \Rightarrow \text { GOTO } 4 \\
& \text { else } \\
& \text { damage } \\
& \downarrow \\
& (\Delta d)_{i}^{(j, k)}=(\Delta \mu)_{i}^{(j, k)} \cdot\left(\frac{\partial G}{\partial \bar{\sigma}}\right)_{i}^{(j, k)} \\
& (d)_{i}^{(j, k)}=(d)_{i}^{(j, k-11}+(\Delta d)_{i}^{(j, k)}
\end{aligned}
$$

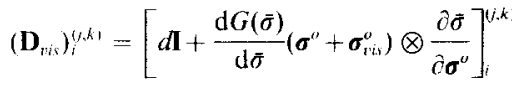

$$
\begin{aligned}
& \left(\mathbf{C}_{i j i s}^{D}\right)^{(j, k)}=\left[\left(\mathbf{I}-\mathbf{D}_{z i s}\right) \mathbf{C}^{v}\right]^{(j, k)} \\
& k=k+1 \text { Go back to } \circledast
\end{aligned}
$$

4. Compute the visco-elastic part of the stresses and the total stresses

$$
\begin{gathered}
\left(\boldsymbol{\eta}^{s}\right)_{i}^{(j, k)}=\alpha\left(\mathbf{C}^{s}\right)_{i}^{(j, k)} \\
\left(\boldsymbol{\sigma}_{i o t}\right)_{i}^{(j)}=(\boldsymbol{\sigma})_{i}^{(j)} \mid\left(\boldsymbol{\eta}^{s} \boldsymbol{\varepsilon}\right)_{t}^{(j, k)}
\end{gathered}
$$

STOP

of $0.175 \mathrm{~g}$ and (b) subjected to the same accelerogram with doubled amplitudes. This allows the simulation of the structural behaviour firstly in a less damaged state [Fig. 7(a)] and finally in a generally collapsed state [Figs 7(b) and 8].

Figures $8(a)$ and $8(\mathrm{~b})$ show the distribution of the sectional damage as given by eqn (56). The damage is located at the joints of the columns with the floors, this being precisely the expected damage localization for this type of structure and load. As the frame is to fail mainly by damage of the columns at their joint with the base floor, the damage plots confirm this prognosed behaviour too. The results of Fig. 9(a) correspond to the undamped case, while in those of Fig. 9(b) the damping effects are included through a value for the relaxation time $\alpha=0.001 \mathrm{~s}$.

The results of Fig. 9 show that the maximum sectional damage $D_{p}$ at the base of the columns is practically equal to the global damage of the entire structure $D$. This fact ratifies the choice of the global damage index as the ratio between the potential energy which the structure cannot undertake in the damaged state and the potential energy that the structure should undertake if it were undamaged. The first floor damage is slightly higher than the global damage of the structure as this floor is the most affected, while the second and third floors follow in decreasing order as the damage reduces with height. The effect of viscous damping is reducing amplitudes and damage levels (Fig. 9(b) and 10). This is in agreement with the well-known real behaviour of structures in a dynamic environment, where the materials display increased strengths and nonconservative energy dissipation. Nevertheless, 


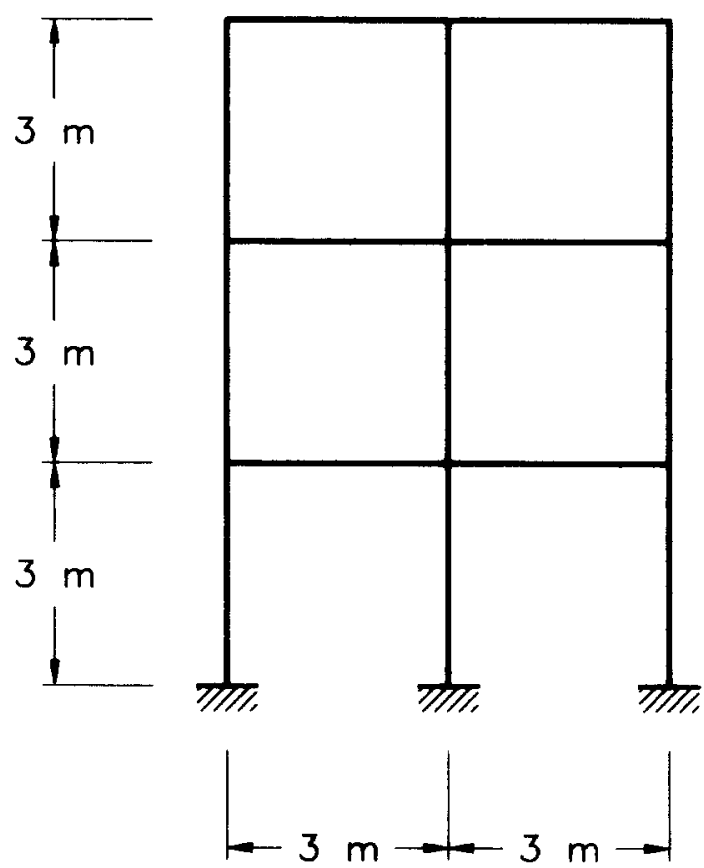

Fig. 5. Geometry of the studied frame.

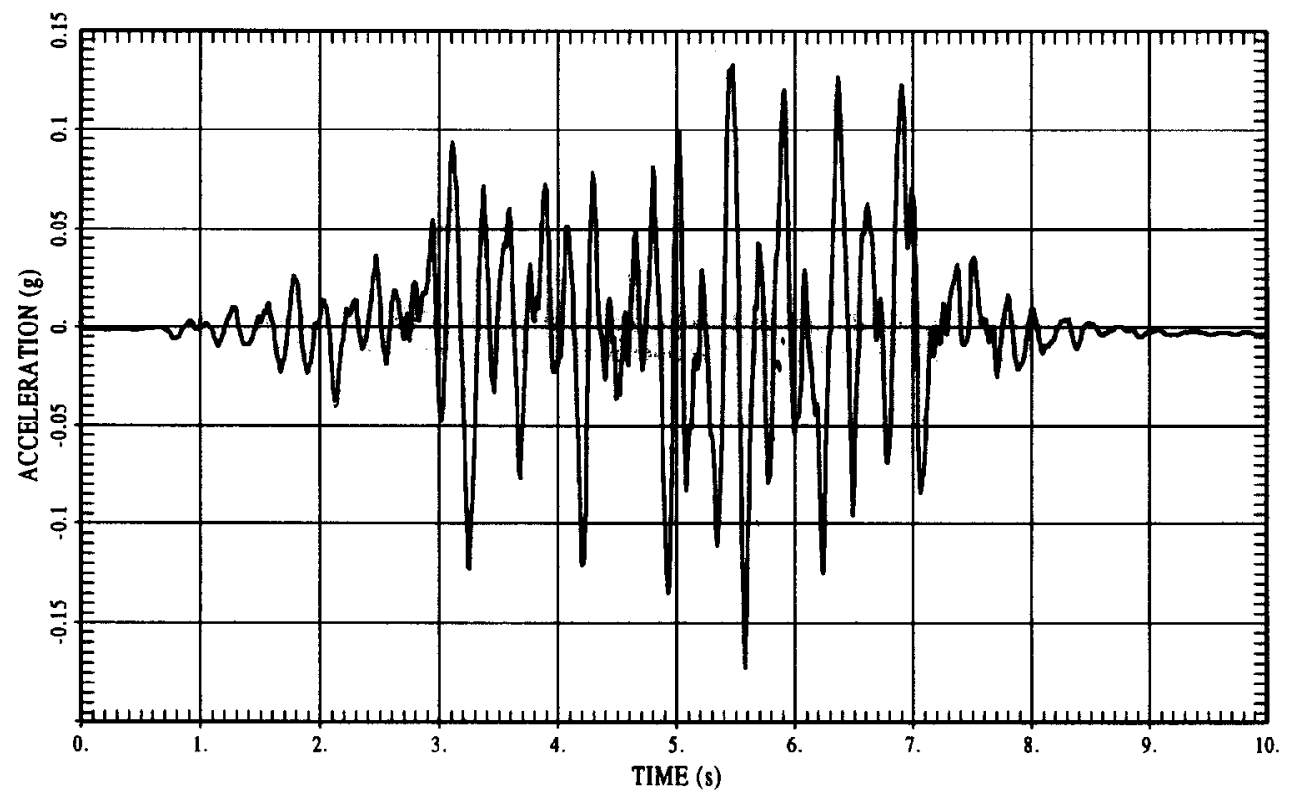

Fig. 6. Synthetic seismic accelerogram corresponding to case (a).

it is necessary to note that, in the simulation process, the damping and the damage have their origin in the material definition but not in the imposed external actions.

\section{Example 2}

The described methodology has been also used to simulate the behaviour of a 3D frame subjected to the same synthetic accelerogram of Fig. 6, acting in the $x$ direction.

The frame has two floors, is $6 \mathrm{~m}$ high and has a squared base of $6 \mathrm{~m}$. The columns have a $30 \mathrm{~cm} \times 30 \mathrm{~cm}$ squared reinforced concrete cross-section. The horizontal beams are $30 \mathrm{~cm}$ thick and $15 \mathrm{~cm}$ wide. All the bars have $8 \%$ of steel, located symmetrically at the corners, with a concrete cover of $3 \mathrm{~cm}$. The density of the concrete has been increased, to take into account the effect of the inertia of the entire floor. The beams placed at one of the sides of 


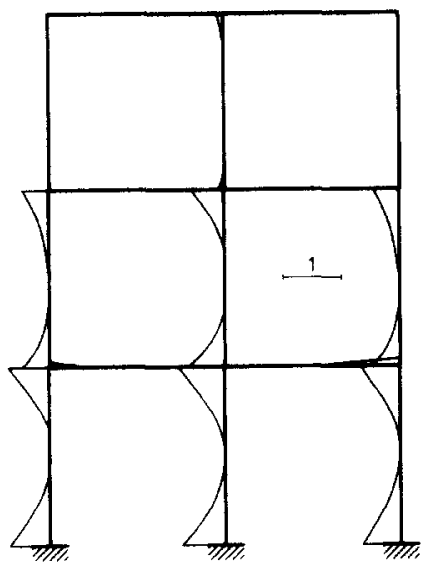

(a)

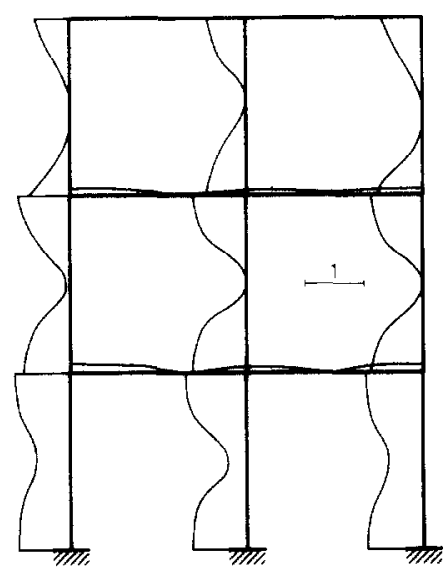

(b)

Fig. 7. Distribution of sectional damage $D_{p}$ all over the structure. Case (a), accelerogram with an amplitude of $0.175 \mathrm{~g}$. Case (b), accelerogram with an amplitude of $0.35 \mathrm{~g}$.

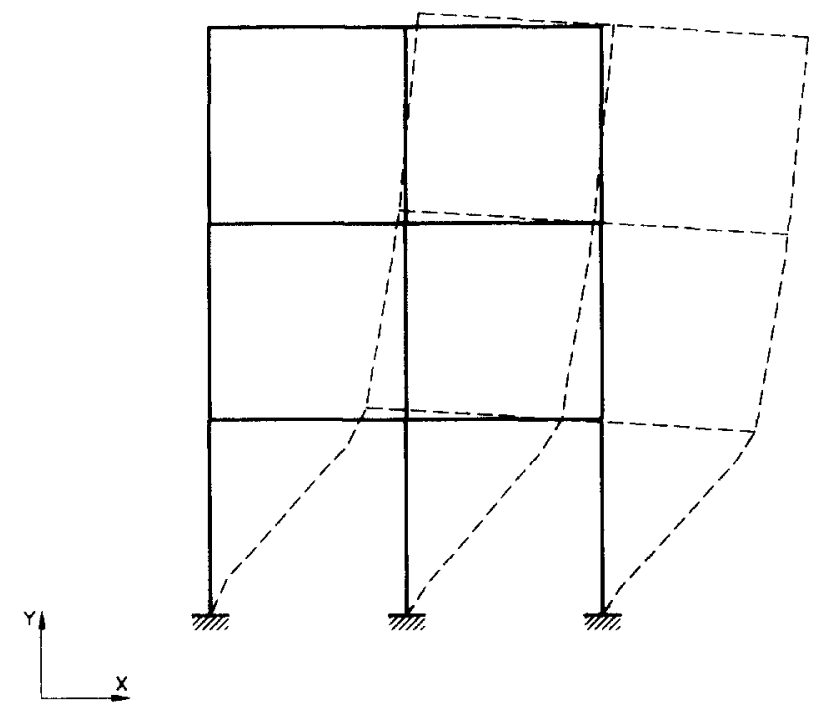

Fig. 8. Deformed configuration at collapse. 

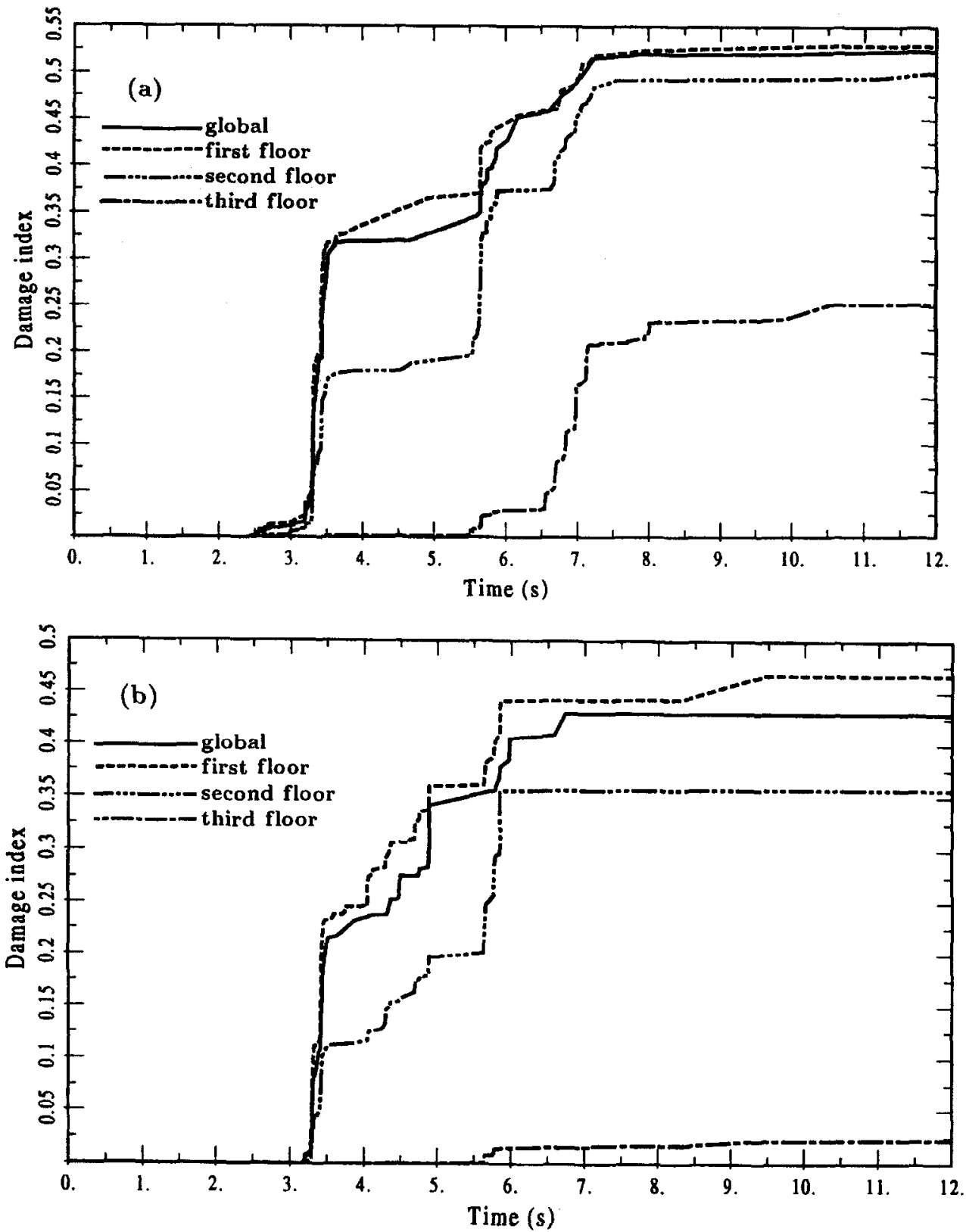

Fig. 9. Global and floor damage indices. Case (a), damping not considered. Case (b), damping considered, relaxation time $\alpha=0.001 \mathrm{~s}$.

the structure (vertical plane $y=6 \mathrm{~m}$ ) have double density, thus the effect of the global structural torsion being simulated.

The structure was discretized in 48 quadratic three-noded beam finite elements with two Gauss points each and the resulted dynamic model has 92 nodes with six degrees of freedom per node. The elements corresponding to the columns are one metre long and those corresponding to the beams are two metres long. All the cross-sections are discretized by means of a $10 \times 10$ grid, appearing thus 400 grid corners per Gauss point at which the state of the material is checked. The materials have the same properties as in Example 1.

The equations of motion have been solved using Newmark's step by step algorithm for $\beta=0.25$ and $\gamma=0.5$. Four deformed shapes of the structure during the earthquake can be seen in Fig. 11. Figure 12 shows the distribution of the sectional damage in the structural elements. The beams are damaged first, due to their higher inertia and smaller stiffness. The columns are highly damaged at their lower part, as expected. It can be observed in Fig. 13 


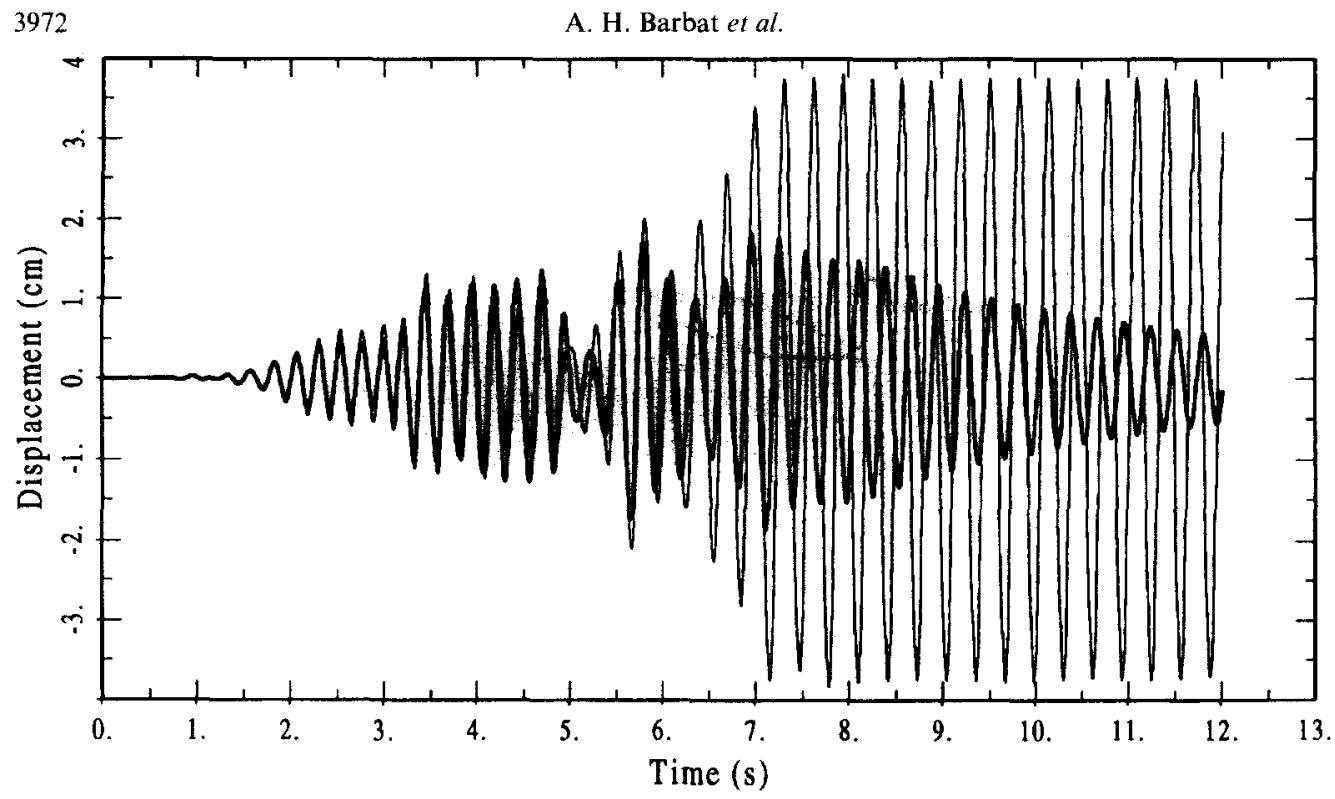

Fig. 10. Third floor displacements, with and without damping.

(a)

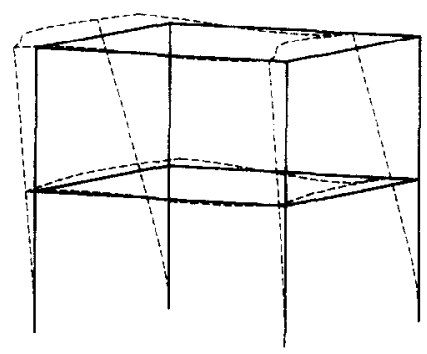

(c)

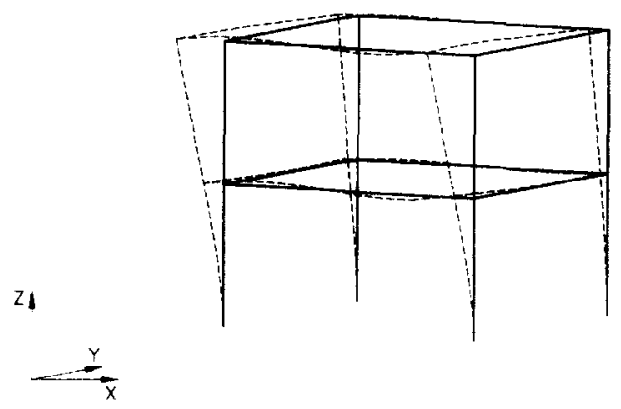

(b)

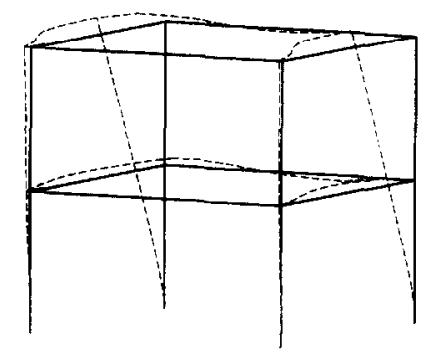

(d)

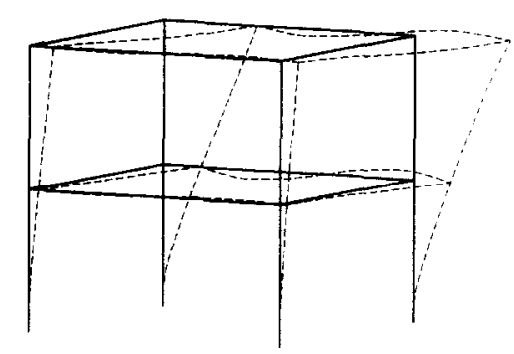

Fig. 11. Deformed shapes of the frame at different time instants during the earthquake.

that both floors are damaged almost simultaneously and that the three compared damage indices have a similar time evolution.

The computational effort required to solve the examples 1 and 2 is cvaluatcd in number of multiplication operations per element and iteration. This number does not take into account the cost of solving the linear systems of equations, but only the evaluation of the residual forces. For Example 1, which uses layered Timoshenko beam elements, this number is of the order of 28,000 . The computer time required to solve completely this example was of half an hour in a CONVEX C3 computer. For Example 2, that is for the 3D Timoshenko beam elements, the number of operations was 144,000 and the computer time used in 
(a)

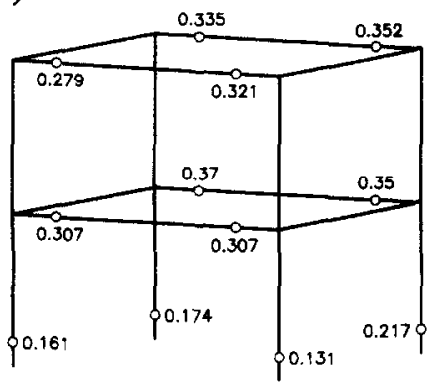

(c)

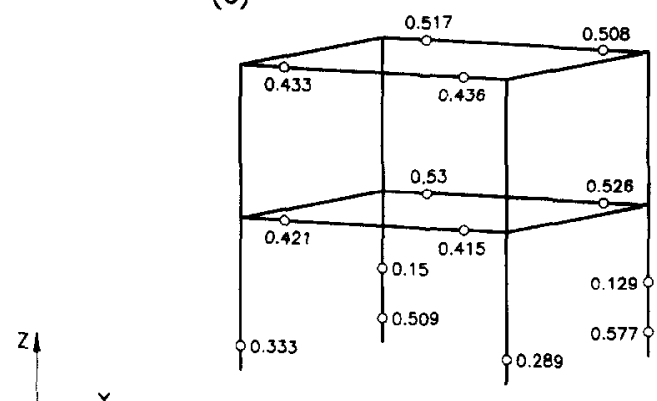

(b)

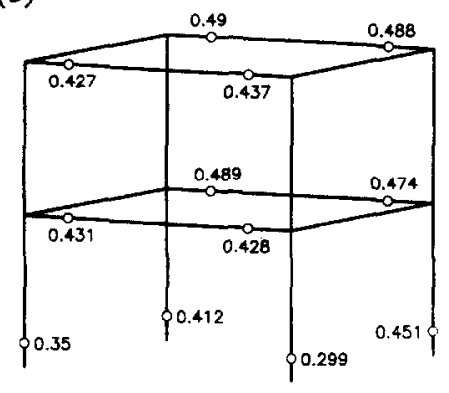

(d)

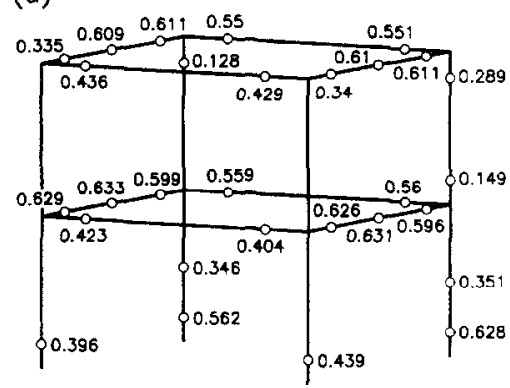

Fig. 12. Evolution of the structural damage level.

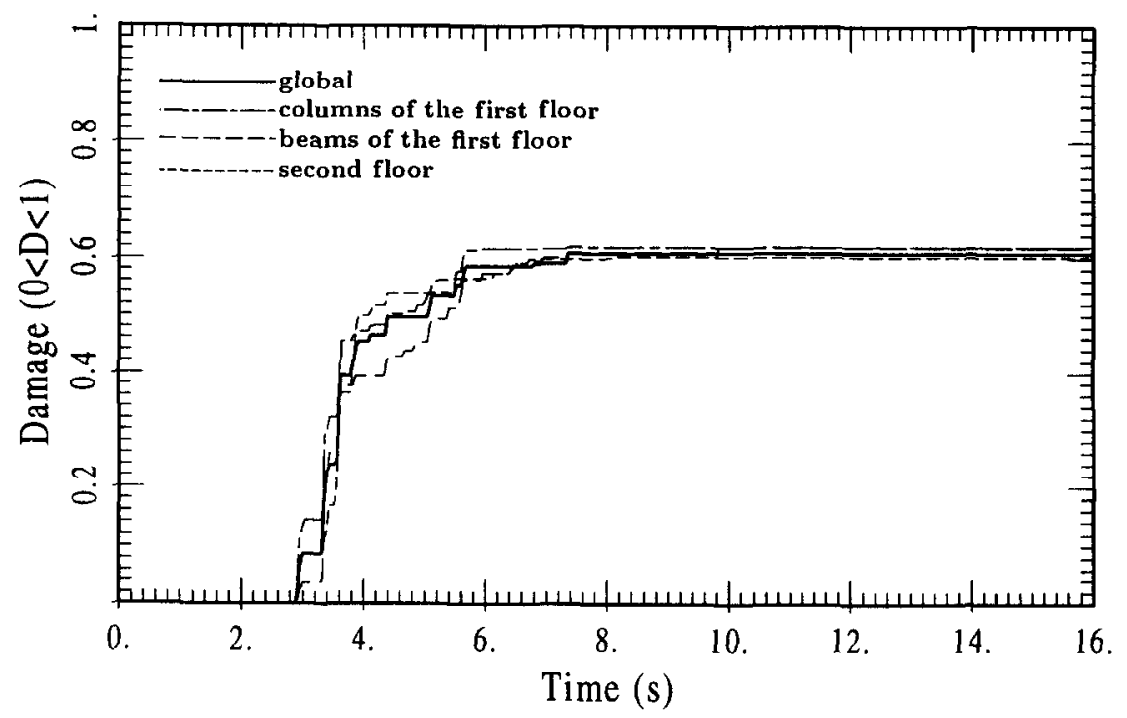

Fig. 13. Evolution of the global and floor damage indices.

solving the complete problem was 7 hours in a CONVEX C3 computer. Note that for 3D hexahedrical elements with 20 nodes, the number of operations would be 3.3 times higher.

\section{Example 3}

The object of this example is the comparison of results obtained by using the damage constitutive model described in this paper with the results of a quasi-static laboratory test performed by Vecchio and Emara (1992) on a reinforced concrete frame. A numerical simulation of the behaviour of the tested frame, but using an elasto-plastic constitutive model, has been already performed by Oller et al. (1996). A complete description of the 
a)

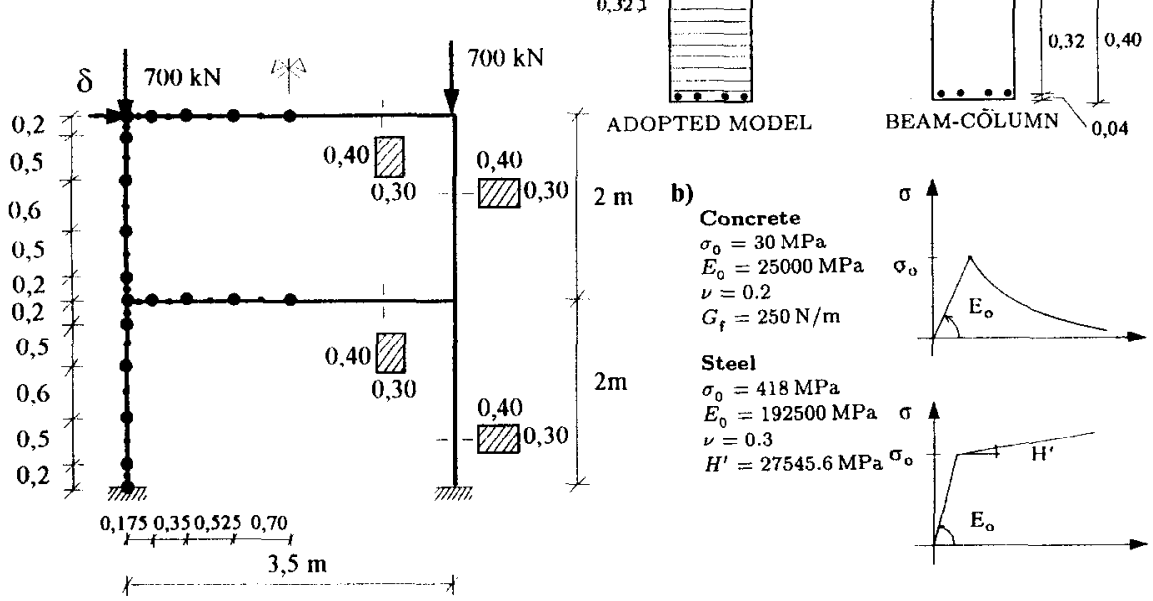

Fig. 14. Description of the geometrical and mechanical characteristics of the frame of Example 3.

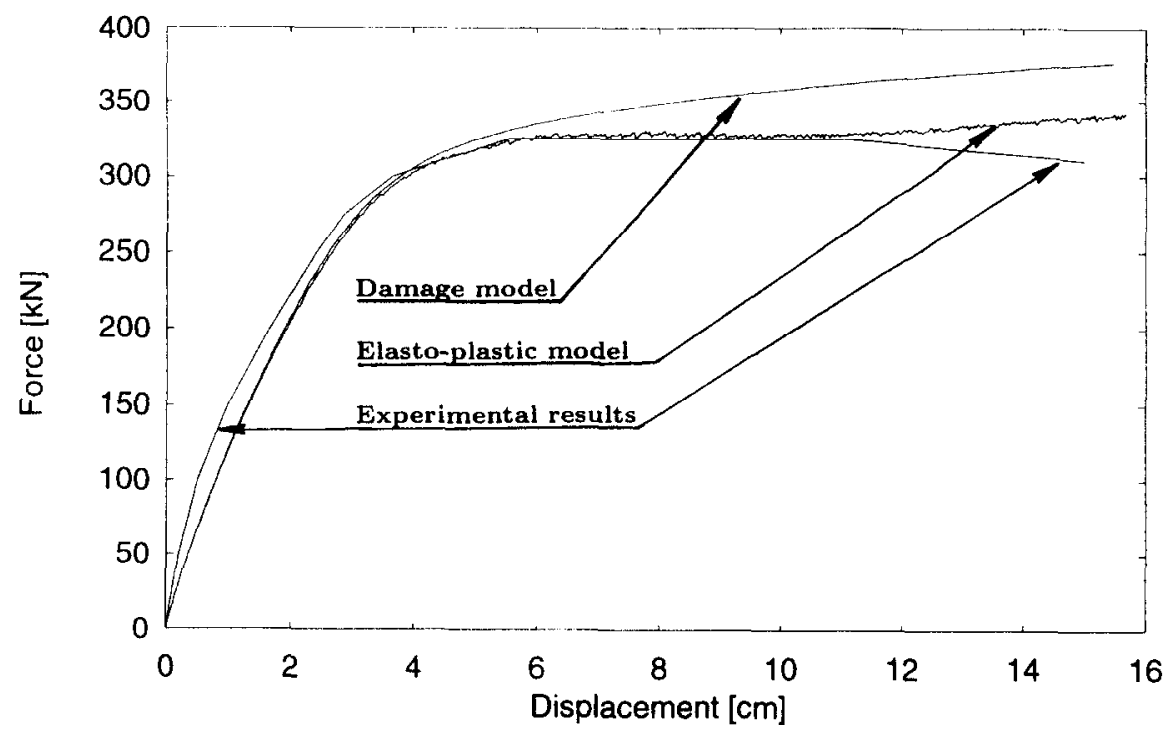

Fig. 15. Comparison of experimental results with results obtained by using the proposed model and an elasto-plastic model.

geometrical and mechanical characteristics of the frame, as well as of the loads, is given in Fig. 14

The laboratory test consisted in applying monotically increasing vertical loads of the two columns (see Fig. 14), which produced thus their pre-compression. This effect had as a result that the damage appeared in the horizontal beams. A horizontal force has been applied afterwards on the beam of the second floor. This force has been increased until the structural failure occurred. The curves in Fig. 15 relate the horizontal forces and displacements for the beam of the second floor and correspond to the laboratory test case and to the computer simulation using an elasto-plastic model (Oller et al., 1996) and the damage model proposed in the present paper. Good agreement between the experimental and simulated results can be observed. Although the elasto-plastic model fits better the experimental results, its much higher computational cost (approximately twice) makes adequate the use of the damage model. 
The simplified visco-damage constitutive model developed has proved to have good performance in describing the nonlinear behaviour of reinforced concrete building structure under dynamic load. The model has been incorporated in a finite element scheme using $2 \mathrm{D}$ and 3D Timoshenko beam elements discretized in a grid of rectangles of concrete and steel in order to approximate the nonlinear behaviour of reinforced concrete beams. A global damage index was deduced from the local damage index supplied by the constitutive model.

A reinforced concrete building structure, under both non viscous and viscous regimes, subjected to seismic actions, has been solved and satisfactory results were obtained. It is shown that the effect of considering the viscosity is of great importance. An interesting property of the global damage index is that of allowing the decision of the state of the structure in what regards its failure mechanisms. The model permits the identification of the mechanism of collapse by observing the local damage indices and continuous comparison with the global one. When, during a damaging process, the global index gets close to the maximum local damage and the rest of the points of the structure stop degrading, the critical points of the structure has been identified. The failure of these points leads to the formation of a failure mechanism, i.e., collapse of the structure. This is important from an engineering structural retrofitting point of view.

The model, in its present form, has two major drawbacks : first, it does not provide information about permanent deformation, which is a well-known feature of non-linear materials and second, it does not discriminate between traction and compression damage, thus being unable to simulate "crack closure". These two problems are currently under study and solutions are already in sight.

\section{REFERENCES}

Aoyama, H. and Sugano, T. (1968) A generalized inelastic analysis of reinforced concrete structures based on tests on members. In Recent Researches of Structural Mechanics. Tokyo.

Barbat, A. H., Hanganu, A., Oller, S. and Onate, E. (1993) Rigorous damping matrix for finite element models with viscous behaviour applied to seismic problems. Computing $5,1-4$.

Barbat, A. H. and Miquel Canet, J. (1989) Structural Response Computation in Earthquake Engineering, Pineridge Press, Swansea.

Bracci, J. M., Reinhorn, A. M., Mander, J. M. and Kunath, S. K. (1989) Deterministic model for seismic damage evaluation of reinforced concrete structures. National Center for Earthquake Engineering Research, Technical Report NCEER-89-0033, State University of New York at Buffalo.

Chen, W. F. (1982) Plasticity in Reinforced Concrete. McGraw-Hill, New York.

Clough, R. W., Benuska, K. L. and Wilson, E. L. (1965) Inelastic earthquake response of tall buildings. In Proceedings of the Third World Conference on Earthquake Engineering, Vol. 2, Auckland, New Zealand, pp. 6889.

DiPasquale, E. and Cakmak, A. S. (1989) On the relation between local and global damage indices. National Center for Earthquake Engineering Research, Technical Report NCEER-89-0034, State University of New York at Buffalo.

Gere, J. and Timoshenko, S. (1984) Mechanics of Materials, 2nd ed. PWS Publishers, New York

Kachanov, L. (1958) Time of rupture process under creep conditions. Izvestia Akademii Nauk 8, 26-31 (in Russia). Lubliner, J. (1990) Plasticity Theory. Macmillan Publishing Company, New York.

Lubliner, J., Oliver, J., Oller, S. and Oñate, E. (1989) A plastic-damage model for concrete. International Journal of Solids and Structures 25(3), 299-326.

Luccioni, B., Oller, S. and Barbat, A. H. (1995) Simulación del amortiguamiento estructral de Rayleygh por medio de modelos viscosis. In XXVII Jornadas Sudamericanas de Ingenieria Estrucutral, Tucuman, Argentina, IV, 1-12.

Massonet, Ch. and Save, M. (1966) Cálculo plástico de las construciones. Montaner y Simon S.A., Barcelona.

Mazars, J. (1991) Damage models for concrete and their usefulness for seismic loadings. In Experimental and Numerical Methods in Earthquake Engineering, eds J. Donea and P. M. Jones. ECSC, EEC, EAEC, Brussels and Luxemburg, pp. 199-221.

Malvern, L. (1969) Introduction to the Mechanics of a Continuous Medium. Prentice Hall, Englewood Cliffs.

Oliver, J., Cervera, J., Oller, S. and Lubliner, J. (1990) Isotropic damage models and smeared crack analysis of concrete. In Proceedings 2nd ICCAADCS, Vol. 2. Zell Am See, Austria, Pineridge Press, pp. 945-958.

Oller, S. (1988) Un modelo de daño continuo para materiales friccionales, Ph.D. thesis (in Spanish). Technical University of Catalonia, Barcelona, Spain.

Oller, S., Oliver, J., Cervera, M. and Oñate, E. (1990) Simulación de un proceso de localización en mecánica de sólidos mediante un modelo plástico. In Proceedings I Congreso Español de Métodos Numéricos. Canarias, SEMNI, pp. 423-431.

Oller, S., Oñate, E., Oliver, J. and Lubliner, J. (1990) Finite element nonlinear analysis of concrete structures using a plastic-damage model. Engineering Fracture Mechanics 35, 219-231. 
Oller, S., Luccioni, B. and Barbat, A. H. (1996) Un método de evaluación del daño sísmico en estructuras de hormigón armado. Revista Internacional de Métodos Numéricos en la Ingenieria 12(2), 215-238.

Oñate, E. (1992) Cálculo de estructuras por el método de los elementos finitos. Centri Internacional de Métodos Numéricos en Ingeneiría, CIMNE, Barcelona.

Park, Y.-J., Ang, A. H.-S. and Wen, Y. K. (1987) Damage limiting aseismic design of buildings. Earthquake Spectra 3(1), 1-26.

Simó, J. C. and Hughes, T. (1995) Elasto Plasticity, Computational Aspects. Springer Verlag, Berlin (to appear).

Simó, J. C. and Ju, J. (1987) Strain and stress based continuum damage models-part I : formulation. International Journal of Solids and Structures 23(7), 281-301.

Vecchio, F. J and Emara, M. B. (1992) Shear deformations in reinforced concrete frames. ACI Structural Journal $89(1), 45-46$. 